This document is confidential and is proprietary to the American Chemical Society and its authors. Do not copy or disclose without written permission. If you have received this item in error, notify the sender and delete all copies.

\title{
1H NMR-Linked Metabolomics Analysis of Liver from a Mouse Model of NP-C1 Disease
}

\begin{tabular}{|r|l|}
\hline Journal: & Journal of Proteome Research \\
\hline Manuscript ID & pr-2016-00238y.R1 \\
\hline Manuscript Type: & Article \\
\hline Date Submitted by the Author: & n/a \\
\hline Complete List of Authors: & $\begin{array}{l}\text { Ruiz-Rodado, Victor ; De Montfort University Faculty of Health and Life } \\
\text { Sciences, School of Pharmacy } \\
\text { Nicoli, Elena-Raluca; University of Oxford, Pharmacology } \\
\text { Probert, Fay; De Montfort University Faculty of Health and Life Sciences, } \\
\text { School of Pharmacy; University of Oxford, Pharmacology } \\
\text { Smith, David; University of Oxford, Pharmacology } \\
\text { Morris, Lauren; University of Oxford, Pharmacology } \\
\text { Wassif, Christopher; National Institute of Child Health and Human } \\
\text { Development } \\
\text { Platt, Frances; University of Oxford, Pharmacology } \\
\text { Grootveld, Martin; De Montfort University, Leicester, School of Pharmacy }\end{array}$ \\
\hline \hline
\end{tabular}


For submission to Journal of Proteome Research

\section{${ }^{1} \mathrm{H}$ NMR-Linked Metabolomics Analysis of Liver from a Mouse Model of NP-C1 Disease}

Victor Ruiz-Rodado ${ }^{1}$, Elena-Raluca Nicoli ${ }^{2}$, Fay Probert ${ }^{1}$, David A. Smith ${ }^{2}$, Lauren Morris ${ }^{2}$, Christopher A. Wassif ${ }^{2,3}$, Frances M. Platt ${ }^{2}$ and Martin Grootveld ${ }^{1^{*}}$.
${ }^{1}$ Leicester School of Pharmacy, De Montfort University, The Gateway, Leicester LE1 9BH, UK.

${ }^{2}$ Department of Pharmacology, University of Oxford, Mansfield Road, Oxford OX1 3QT, UK.
${ }^{3}$ Eunice Kennedy Shriver National Institute of Child Health and Human Development, NIH, Bethesda, USA.

*Author for correspondence.

Email: mgrootveld@dmu.ac.uk

Tel.: +0044-(0)161-250-6443 


\begin{abstract}
Background/Aims: Clinical manifestations of Niemann Pick type-C1 (NP-C1) disease include neonatal hepatosplenomegaly, and in some patients progressive liver dysfunction and failure. This study involved a ${ }^{1} \mathrm{H}-\mathrm{NMR}$ linked metabolomics analysis of liver samples collected from a NP-C1 disease mutant mouse model in order to explore time-dependent imbalances in metabolic pathways associated with NP-C1 liver dysfunction, including fibrosis.
\end{abstract}

Methods: NP-C1 mutant (Npc1 $\left.1^{-/} ; \mathrm{NP}-\mathrm{C} 1\right)$, control $\left(\mathrm{Npc}^{+/+} ; \mathrm{WT}\right)$ and NP-C1 heterozygous mice $\left(\mathrm{Npc1}^{+/-} ; \mathrm{HET}\right)$ were generated from heterozygote matings. Aqueous extracts of these liver samples collected at time-points of 3, 6, 9 and 11 weeks were subjected to highresolution NMR analysis, and multivariate (MV) metabolomics analyses of datasets acquired were performed.

Results: A MV random forests (RFs) model effectively discriminated between the NP-C1 and a combined $\mathrm{HC} / \mathrm{HET}$ hepatic NMR profiles with very high predictive accuracy and reliability. Key distinguishing features included significant upregulations in the hepatic concentrations of phenylalanine, tyrosine, glutamate, lysine/ornithine, valine, threonine, and hypotaurine/methionine, and diminished levels of nicotinate/niacinamide, inosine, phosphoenolpyruvate and 3-hydroxyphenylacetate. Quantitative pathway topological analysis confirmed that imbalances in tyrosine biosynthesis, hepatic phenylalanine, tyrosine, glutamate/glutamine and nicotinate/niacinamide metabolism were involved in the pathogenesis of NP-C1 disease-associated liver dysfunction/damage.

Conclusions: ${ }^{1} \mathrm{H}$ NMR-linked metabolomics analysis provides valuable biomarker information regarding hepatic dysfunction or damage in NP-C1 disease.

Keywords: NP-C1 Disease; Liver Damage/Dysfunction; Fibrosis-cirrhosis; NMR-Based Metabolomics; Biomarkers; Amino Acid Biosynthesis and Metabolism; Nicotinate and Niacinamide Metabolism; 3-Hydroxyphenylacetate; Metabolic Pathway Analysis. 


\section{INTRODUCTION}

Niemann-Pick type C (NP-C) disease is a neurodegenerative lysosomal storage disorder caused by mutations in the NPC1 or NPC2 genes, the hereditability of which is autosomal recessive. ${ }^{1,2}$ Approximately $95 \%$ of patients have mutations in the NPC1 gene (mapped to 18q11) which encodes a large membrane glycoprotein primarily located in late endosomes (LEs) and lysosomes. ${ }^{3}$ The remaining $5 \%$ of mutations are in the NP-C2 gene (mapped to 14q24.3), which encodes a small soluble lysosomal protein that binds cholesterol in the lysosomal lumen. ${ }^{3}$ Patients affected by NP-C1 or NP-C2 diseases are indistinguishable, both clinically and in terms of any standard biochemical evaluations available for NP-C disease diagnosis. $^{4}$

Dysfunction of NPC1 or NPC2 proteins leads to a complex storage pattern of cholesterol and sphingolipids in late endosomes/lysosomes. Progressive neurodegeneration, together with liver and lung disease are characteristic symptoms..$^{5}$ Liver disease features, such as cholestasis with neonatal jaundice, together with hepatosplenomegaly, are usually encountered in the early stages of the disease; indeed, in some patients these symptoms become chronic and hence fatalities from liver failure occur. ${ }^{6}$ The pathophysiology associated with NP-C1 disease-associated liver dysfunction are of much importance, since this organ plays a central role in maintaining whole-body cholesterol and lipoprotein metabolism. ${ }^{7}$ Several studies in NP-C1 mice suggest that hepatocyte apoptosis may represent a primary cause of liver dysfunction and failure. ${ }^{8,9}$ Moreover, an increased activation of macrophages serves as a further cause of progressive liver degeneration in this animal model. $^{10}$ 
Metabolic profiling of biofluids and/or tissues by high-resolution NMR analysis has been successfully applied to explore the pathogenesis of a wide range of clinical conditions, ${ }^{11-13}$ and recent investigations have demonstrated the utility of this technique for the investigation of NP-C disease. ${ }^{14,15}$ Moreover, a multivariate genomics approach has been employed for seeking biomarkers for NP-C1 disease in serum. ${ }^{16}$ NMR-linked metabolomics analysis can provide a complete evaluation of low-molecular-mass metabolites present in the hepatic tissue of experimental animal models, and in this context offers potential for the provision of valuable metabolic profiling information. ${ }^{17}$ This strategy offers major advantages over many conventional bioanalytical approaches for the evaluation of liver disease, which provide information regarding the concentrations of perhaps only several biomarkers, and which are therefore insufficient for the provision of a global metabolic characterisation for comparative classification purposes.

In this investigation, we report for the first time a ${ }^{1} \mathrm{H}$ NMR-linked metabolomics investigation of the biomolecular profiles of liver specimens collected from NP-C1 diseaseactive, age-matched heterozygous NP-C1 carrier, and healthy control mice in an established animal model system. ${ }^{18}$ For this purpose, we employed a cross-validated Random Forests (RFs) model, together with further multivariate (MV) analysis strategies. Npc1-null mutant mice $\left(\mathrm{Npc1}^{-/-}\right)$serve as a valuable model system for exploring the impact of $\mathrm{Npc1}$ deficiency, and features a range of the biochemical, pathological, neurological and behavioural characteristics of human NP-C disease (these include the storage of unesterified cholesterol and sphingolipids, Purkinje cell loss, axonal swelling, neuronal vacuoles and progressive motor impairments such as tremor, ataxia, and loss of co-ordination; ${ }^{19-21}$ subsequent to a 
progressive body weight loss, these mice die at ages of $10-12$ weeks $^{20}$ ). In order to investigate the onset and progression of hepatic pathology in Npc1-deficient mice, liver samples of NP-C1 mutant mice, and age-matched heterozygous carriers, and controls, were examined at 4 time-points during their life span, i.e. those corresponding to presymptomatic ( 3 weeks), early symptomatic ( 6 weeks) and late symptomatic ( 9 weeks) and end stage (11 weeks) criteria.

The biochemical and metabolic significance of the results acquired were further analysed and evaluated via the application of quantitative metabolite set enrichment and metabolic pathway topological analysis. The potential clinical significance of the results in relation to NP-C1-associated liver dysfunction are discussed in detail.

\section{MATERIALS AND METHODS}

2.1 Experimental animals: A spontaneous mutant mouse model of NPC on a BALB/C

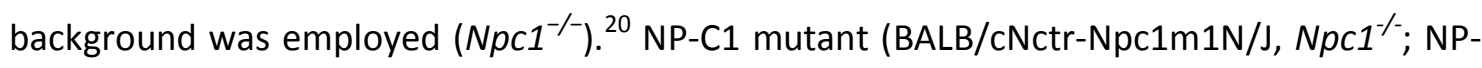
$\mathrm{C1})$, control $\left(\mathrm{Npc1}^{+/+} ; \mathrm{WT}\right)$ and NP-C1 heterozygous mice $\left(\mathrm{Npc1}^{+/-} ; \mathrm{HET}\right)$, were generated from heterozygote matings. The wild-type littermates $(+/+)$ were used as controls. Genotyping of NP-C1 mice was determined by polymerase chain reaction (PCR) as described previously. ${ }^{22}$ Mice were bred and housed under standard sterile conditions at the University of Oxford. All animal procedures were conducted using protocols approved by the UK Animals (Scientific Procedures) Act (1986). The $N p c 1^{-/-}$mice have a lifespan of 10-12 weeks (mean 10.5 weeks), with neurological symptoms presenting from 5-6 weeks of age. In 
total, 89 liver samples from gender-matched heterozygous carrier (HET), NP-C1 disease (NPC1) and WT mice were collected at time-points of 3, 6, 9 and 11 weeks (Table 1).

\subsection{Extraction of aqueous metabolites for ${ }^{1} \mathrm{H}$ NMR-linked metabolomics analysis: The} extraction protocol employed was based on the procedure described by Waters et al. ${ }^{23} \mathrm{~A}$ portion of hepatic tissue ( $c a .80 \mathrm{mg}$ ) from each liver sample was accurately weighed, and an ice-cold extraction solvent $\left(1: 1 \mathrm{H}_{2} \mathrm{O}: \mathrm{CH}_{3} \mathrm{CN}\right)$ was then added at a volume equivalent to $20 \mu \mathrm{L}$ per mg of tissue. Subsequently, the sample was mechanically homogenised using an electric pestle rotor (Sigma-Aldrich, UK), and the homogenates were then centrifuged at $10,000 \times g$ for a period of $10.0 \mathrm{~min}$. at $4^{\circ} \mathrm{C} .1 .00 \mathrm{~mL}$ volumes of each supernatant (containing hydrophilic metabolites) were lyophilised and then reconstituted in $500 \mu \mathrm{L}$ of $\mathrm{D}_{2} \mathrm{O}$ containing $0.05 \%(\mathrm{w} / \mathrm{v})$ sodium 3 -trimethylsilyl-(2,2,3,3- $\left.{ }^{2} \mathrm{H}_{4}\right)$-1-propionate (TSP, chemical shift reference and internal quantitative ${ }^{1} \mathrm{H}$ NMR standard) and $50 \mu \mathrm{L}$ of a $1.00 \mathrm{M}$ solution of phosphate buffer $(\mathrm{pH}$ 7.00). Subsequently, samples were thoroughly rotamixed and centrifuged at 5,000 $\times \mathrm{g}$ for $5.0 \mathrm{~min}$. at ambient temperature, and the clear supernatants transferred to 5-mm NMR tubes for analysis.

2.3 ${ }^{1} \mathrm{H}$ NMR analysis: Single-pulse ${ }^{1} \mathrm{H}-\mathrm{NMR}$ spectra were acquired on a Bruker Avance AM400 spectrometer (Leicester School of Pharmacy facility, DMU, Leicester, UK) operating at a frequency of $399.94 \mathrm{MHz}$ and a probe temperature of $298 \mathrm{~K}$. These spectral acquisitions involved the noesygppr1d (TOPSPIN version 3.0, Bruker Biospin) pulse sequence for water suppression. For each spectrum, 128 scans were acquired, with a relaxation delay of $3 \mathrm{~s}$, a spectral width of $4,800 \mathrm{~Hz}$ and a time domain of $32 \mathrm{~K}$ points. Spectra were acquired in an automated manner using a sample changer for continuous sample delivery. 
Spectra were phased and baseline-corrected using ACD/labs Spectrus Processor 2013, and an exponential line-broadening function of $0.30 \mathrm{~Hz}$ was applied. Subsequently, the intelligent bucketing procedure was employed, giving a dataset of 89 rows (samples) and 175 columns (potential predictor variables). The resulting intelligently-selected bucket (ISB) integral intensity values were normalised to the TSP internal standard signal $(s, \delta=0.00$ ppm). Spectral regions which did not contain any resonances were removed, yielding a final dataset of 89 (samples/rows) $\times 136$ (variables/columns) for further analysis. The intense $\delta=$ 4.65-5.16 ppm residual $\mathrm{H}_{2} \mathrm{O} / \mathrm{HOD}$ signal region was also removed from all the ${ }^{1} \mathrm{H} N M R$ profiles acquired prior to the performance of MV data analysis strategies.

2.4 Metabolite Identification: ${ }^{1} \mathrm{H}-\mathrm{NMR}$ spectra were assigned on the basis of literature values, ${ }^{17,24}$ available databases (HMDB), ${ }^{25}$ and by the consideration of chemical shift values, coupling patterns and coupling constants. The two-dimensional (2D) NMR techniques ${ }^{1} \mathrm{H}-{ }^{13} \mathrm{C}$ HSQC (heteronuclear single quantum correlation) and ${ }^{1} \mathrm{H}-{ }^{1} \mathrm{H}$ COSY (correlation spectroscopy were employed for structural confirmation purposes. HSQC and COSY spectra were acquired on the above Bruker Avance AM-400 spectrometer. For the HSQC experiments, the hsqcedetgpsp.3 pulse sequence (TOPSPIN version 3.0, Bruker Biospin) was employed, and 256 scans for 128 increments were acquired for each spectrum. The F2 and F1 spectral widths were 6,340 and $16,600 \mathrm{~Hz}$, respectively. COSY spectra were acquired using the cosygpprqf pulse sequence (TOPSPIN version 3.0, Bruker Biospin), recording 48 scans. The spectral width was $4,000 \mathrm{~Hz}$. 
2.5 Data Processing, Univariate and Multivariate Analysis: The integral values obtained from the ISB procedure outlined in section 2.3 were cube root-transformed and Paretoscaled in order to satisfy assumptions of normality and homogeneity of intra-sample variances, which is a stringent requirement for the additional statistical analysis strategies subsequently applied, including analysis-of-covariance (ANCOVA), together with ANOVASimultaneous Component Analysis (ASCA) ${ }^{26}$ Nevertheless, since the RFs technique has a non-parametric basis, it is generally unaffected by such feature scaling.

The experimental design for univariate analysis of the ${ }^{1} \mathrm{H}$ NMR ISB intensity datasets involved an ANCOVA model, which incorporated 3 factors and 6 primary sources of variation: (1) 'between-disease classifications' (qualitative NP-C1 disease-active vs. the combined HET/WT control group), fixed effect $\left(D_{i}\right)$; (2) 'between-genders' fixed effect (qualitative) 'nested' within 'disease classifications' $\left(\mathrm{G}_{j}\right)$; $(3)$ the experimental sampling timepoint fixed effect (quantitative, $\left.T_{k}\right) ;(4,5$ and 6$)$ disease classification $x$ gender, disease classification $x$ time-point and gender $x$ time-point first-order interaction components $\left(D_{i j}\right.$, $\mathrm{DT}_{i k}$ and $\mathrm{GT}_{j k}$ ) respectively. This experimental design is represented by equation 1 , in which $\mathrm{Y}_{i j k}$ represents the (univariate) ISB predictor variable values observed, $\mu$ its overall population mean value in the absence of any significant, influential sources of variation, and $\mathrm{e}_{i j k}$ the unexplained error (residual) contribution.

$Y_{i j k}=\mu+D_{i}+G_{j}+T_{k}+D_{i j}+D T_{i k}+G_{j k}+e_{i j k}$ 
ANCOVA was conducted with XLSTAT2014. ASCA, principal components analysis (PCA) and partial least-squares discriminatory analysis (PLS-DA) were applied using MetaboAnalyst $3.0 .^{27}$

RFs analysis was employed for classification and variable selection purposes using the RandomForest $\mathrm{R}$ package. ${ }^{28}$ RFs analysis was applied over 100 iterations. For each iteration, the mean and standard error (SEM) of the out-of-the-bag (OOB) error, and confusion matrix were computed. For each of the 100 iterations performed $33 \%$ of the samples were included in the test-set, whilst the remaining $67 \%$ served as the training-set. For variable selection purposes, the variable importance parameter values for the 136 variables were estimated using the mean decrease in accuracy (MDA) value, since this index has been suggested to be the most reliable metric for this purpose. ${ }^{29,30}$ Further details regarding the RFs approach employed here are available in section S1.

The RFs model was tuned for the analysis of the NP-C1 vs. the combined Het/WT classifications (based on the OOB error value obtained in order to improve its performance), so that optimal mtry (number of variables selected for splitting at each node involved), and ntree (number of trees) values were selected to be 11 and 1,000 respectively.

In order to evaluate the ability of the RFs technique to classify and predict, a Receiver Operating Characteristic (ROC) curve analysis was also performed using the MetaboAnalyst ROCCET option available. ${ }^{31}$ Further validation was performed with XLSTAT2015, which employed permutation testing via partial redundancy analysis (P-RDA), with gender and sampling time-point as the 'conditioning' variables, and $5 \times 10^{4}$ permutations. 
2.6 Quantitative Metabolic Pathway Topological Analysis: Detailed analysis of the most relevant hepatic metabolic pathways and networks involved in the pathogenesis of NP-C1 disease was performed by MetaboAnalyst 3.0 tools. ${ }^{27}$ For this dataset, we employed the Global ANCOVA and Relative-Betweeness Centrality algorithm for metabolic pathway and topological analyses, the latter serving as a key measure indicating the importance of a graph node when expressed relative to that of the others. ${ }^{32}$ For the quantitative pathway topological analysis performed, the mus musculus (mouse) mammalian pathway library was selected. This approach was also utilised to provide estimates of pathway impact and false discovery rate (FDR)-adjusted $p$ values.

\section{RESULTS}

\section{1 ${ }^{1} \mathrm{H}$ NMR Profiles of liver samples collected from NP-C1 disease, heterozygous carrier} and wild type mice: Typical $400 \mathrm{MHz}{ }^{1} \mathrm{H}$ NMR spectra of aqueous extracts of liver samples collected from NP-C1 disease mice (Figure 1), together with corresponding heterozygous carrier (HET) and healthy control (WT) specimens contained a large number of prominent resonances assignable to a wide range of low-molecular-mass metabolites, including amino acids, organic acid anions, carbohydrates and nucleotides.

The acquisition of $2 \mathrm{D}{ }^{1} \mathrm{H}_{-}{ }^{13} \mathrm{C} \mathrm{HSQC}$ and ${ }^{1} \mathrm{H}-{ }^{1} \mathrm{H}$ COSY NMR techniques readily confirmed the structural identities of many of the resonances present in the $1 \mathrm{D}^{1} \mathrm{H}$ NMR profiles of the liver sample aqueous extracts (a full list is provided in Table S1). In total, greater than 40 metabolites were unambiguously assigned by the employment of combinations of two or more of these $1 \mathrm{D}$ and 2D bioanalytical NMR strategies. 
The assignment of phenylalanine, tyrosine, nicotinate and niacinamide (nicotinamide) was facile since their ${ }^{1} \mathrm{H}$ NMR signals are clearly visible in unobscured regions of the $1 \mathrm{D}{ }^{1} \mathrm{H} N M R$ spectra acquired, and in some cases further supporting evidence for such assignments was obtained, since two or more resonances arising from individual metabolites were indicated as discriminatory variables. However, application of 2D-NMR experiments was required to confirm assignments of the valine resonances since they are located at chemical shift values close to those of leucine and isoleucine. Additionally, both methionine and hypotaurine have triplet resonances located at $c a .2 .65 \mathrm{ppm}$, coupled to a multiplet located at $2.15 \mathrm{ppm}$ for the former, and a triplet at $3.35 \mathrm{ppm}$ for the latter; both these linkages were observed in the 2D COSY NMR experiments conducted (Figure S2). The 3-hydroxyphenylacetate resonance was detectable, at low intensity, in 23 of the HET and WT liver samples, but only in 4 of the NP-C1 samples, specifically the 6.78-6.81 ppm ISB; generally, an uncrowded region for all the tissue spectra acquired. Similarly, the phosphoenolpyruvate (PEP)- $\mathrm{CH}_{2 a}$ resonance was readily visible, as was inosine's purine ring- $\mathrm{C} 2-\mathrm{CH}$ signal $(s, \delta=8.35 \mathrm{ppm})$, which also served as a significant discriminatory feature in the experiments conducted.

Assignments for the aspartate and glutamate resonances were also confirmed by such 2DNMR experiments. The $\mathrm{ABX}$ coupling system of the $\mathrm{C} 2-\mathrm{CH}_{b}$ proton resonance of aspartate lies within the 2.79-2.81 ppm ISB. For the combined lysine /ornithine ISB resonances $(\delta=$ 1.70-1.74 and 2.99-3.05 ppm), ${ }^{1} \mathrm{H}_{-}{ }^{13} \mathrm{C}$ HSQC spectra acquired confirmed the identities of these amino acids, and hence this ${ }^{1}$ H NMR ISB did not arise from further metabolites with similar $\delta$ values (for example, putrescine and spermidine). 
3.2 Preliminary Multivariate Exploratory Data Analysis: Preliminary PCA and crossvalidated (CV) PLS-DA MV analyses conducted revealed that the most important discriminatory metabolic ISB variable features predominantly comprised resonances arising from amino acids and nucleotide metabolites. Indeed, both these forms of analysis revealed that there was a significant clustering of the NP-C1 disease classification which was distinct from both the HET and WT groups; however, no discrimination between the latter two disease status classifications were found. Indeed, with this unsupervised PCA approach, the 3D component 4 vs. component 3 vs. component 2 scores plot [Figure 2(a)] provided the best visualisation of this NP-C1 vs. the combined HET/WT group discrimination. The NP-C1 cluster exhibited lower scores vectors than those of the combined HET/WT ones for components 3 and 4; there appeared to be no 'between-classification' differences between these scores vectors for component 2, as notable from projections of the 3D plot on the 2D planes.

3.3 RFs strategy for classification purposes: As noted above, primarily the 3 classification groups (i.e. NP-C1, HET and WT) were analysed. The RFs MV analysis strategy performed for direct comparisons of the HET vs. WT classifications resulted in an OOB error value of 0.459 $(\mathrm{SEM}=0.00061)$, suggesting no significant differences between these groups. However, significant values for the OOB error term were obtained when the RFs approach was employed for comparisons of the NP-C1 vs. HET, and NP-C1 vs. WT control groups [OOB error values of $0.146(\mathrm{SEM}=0.00019)$ and $0.230(\mathrm{SEM}=0.00023)$ respectively]. For these analyses, the NP-C1 vs. HET group comparison yielded predictive accuracies of 76.2, 79.8, $82.1,84.9,87.4$ and $87.8 \%$ for models with a maximum of $5,10,15,25,50$ and 100 variables respectively (corresponding AUROC values were 0.840, 0.893, 0.913, 0.929, 0.953 and 0.960 
respectively), whereas for the NP-C1 vs. WT group comparison, these results demonstrated predictive accuracies of $75.1,77.2,78.9,80.6,81.6$ and $81.0 \%$ for models with $5,10,15,25$, 50 and 100 variables respectively (corresponding AUROC values were $0.819,0.851,0.866$, $0.885,0.892$ and 0.898 respectively). Hence, there was a marginally improved level of discrimination achieved in the NP-C1 disease vs. HET comparison over that of the NP-C1 vs. WT one (Figure S1).

These results are consistent with those arising from the PCA results described above. In view of these observations, the HET and WT groups were combined (and labelled HET/WT) for further analysis. When applied to these alternative sample groupings, this RFs approach gave an $\mathrm{OOB}$ error value of $0.156 \pm 0.00013$ (mean $\pm \mathrm{SEM}$ ). For predictive performance, values for sensitivity, specificity and accuracy were $0.84 \pm 0.00069,0.89 \pm 0.0017$ and $0.85 \pm$ 0.00050 respectively. The classification performance of this RFs model is exhibited in Figure 2(b).

We also elected to perform the PLS-DA and orthogonal PLS-DA (OPLS-DA) techniques in order to directly compare the 3-week time-point dataset with that of the 9-week one for both the NP-C1 and HET/WT disease classification groups. Indeed, a PLS-DA model featuring 4 sub-groups (specifically the 3- and 9-week time-points only for both the NP-C1 disease and HET/WT control groups) demonstrated that the 9-week (late stage) NP-C1 disease group formed a cluster that was clearly distinct from that of the 3-week (pre-symptomatic) NP-C1 disease one, together with those of both the 3- and 9-week time-points of the HET/WT classifications, the latter two being indistinguishable [Figure 3(a)]. For this approach, a maximum $Q^{2}$ value of 0.534 was obtained for a model featuring 5 components, and model 
validation performed by permutation testing (with 2,000 permutations) based on separation distance gave a highly significant $p$ value of 0.0045 . Key biomarker features responsible for the discrimination of the 9-week NP-C1 disease classification from the 3- and 9-week HET/WT one were the upregulated valine, phenylalanine, threonine resonances, in addition to the combined hypotaurine/methionine one [Variable Importance Parameter (VIP) values ranged from 1.41-1.74].

Similarly, an O-PLS-DA analysis [Figures $3(\mathrm{~b})$ and (c)] confirmed the significance of the upregulated valine, phenylalanine and threonine predictor variables, and also provided supporting evidence for the involvement of downregulated PEP, 3-hydroxyphenylacetate, and the unassigned $0.77-0.80 \mathrm{ppm}$ resonance as significant biomarker features (these predictors were isolated via a scrutinization of the O-PLS-DA S-plot). This O-PLS-DA analysis was also validated through permutation testing $\left(2,000\right.$ permutations), and the $Q^{2}$ and $R^{2} Y$ values of 0.534 and 0.812 were very highly significant $\left(p=5.00 \times 10^{-4}\right.$ for both parameters).

3.4 Variable selection: The top 15-predictor variable features from the RFs analysis, together with their respective rankings and assignments are shown in Table 2. These variables included significantly elevated hepatic spectral intensities of key amino acids (phenylalanine, tyrosine, glutamate, lysine/ornithine, hypotaurine/methionine, threonine and valine), along with reduced ones for nicotinate/niacinamide, 3-hydroxyphenylacetate, inosine, PEP and an unassigned species with a triplet resonance located within the $0.77-0.80$ ppm ISB. Whilst this latter resonance remains unassigned, ${ }^{1} \mathrm{H}-{ }^{1} \mathrm{H}$ COSY NMR analysis demonstrated that this triplet signal was linked to a multiplet centred at $\delta=1.99 \mathrm{ppm}$ (Figure S2), an observation indicating that the latter is attributable to a proton located $\alpha$ - to 
a carboxylate function, i.e. these coupled resonances appear to arise from a carboxylic acid anion species.

Although the unassigned $0.77-0.80 \mathrm{ppm}$ ISB variable was not found to be significant in a univariate context, it should be noted that multivariate (MV) analysis strategies make use of all variables simultaneously, and therefore focus on simultaneous relationships, linear or otherwise, amongst all available variables. Indeed, these approaches focus on covariances/correlations between these variables, i.e. they reflect the extent of these relationships between potentially large numbers of variables, unlike univariate methods which simply consider the mean and variance values of single variables.

\subsection{Investigations of potential 'between-gender' and 'between-sampling time-point'}

variance component contributions: For further investigation of the potential 'betweengender' component of variance contribution to the MV dataset, the RFs procedure outlined above was again applied, but in this case with gender as the classification criterion. This analysis was performed in order to confirm that the gender variance contribution was not significant, i.e. that it did not condition the disease biomarker variables selected. Since the $\mathrm{OOB}$ error value obtained was $0.44(\mathrm{SEM}=0.000423)$ [accuracy $(\mathrm{SEM})=0.54(0.000837)$; sensitivity $(\mathrm{SEM})=0.55(0.00238)$, specificity $(\mathrm{SEM})=0.56(0.00138)]$, this confirmed that the significant metabolite differences observed were not influenced by this variance component.

Collection time-points were investigated using an ASCA time series analysis option. No significant differences were observed 'between-time-points' after performing this analysis 
$(p=0.091)$, although this value was close to attaining statistical significance; furthermore, the 'disease classification $x$ time-point' interaction component of variance was also found not to be significant for the first two (major) PCs explored $(p=0.198)$. However, as expected, the 'between-diseases' factor was indeed significant $(p=0.006)$. Moreover, the P-RDA approach, which involved the removal of any contributory effects exerted by the quantitative time-point and qualitative gender 'conditioning' variables confirmed the significance of the 'between-diseases' variable $(p=0.0032)$. This strategy was also applied to explore the contributions of gender and experimental sampling time-point variables (with the effects exerted by combinations of the disease classification/sampling time-point and disease classification/gender factors, respectively, removed as 'conditioning' variables) revealed that the time-point variable was indeed significant $(p=0.022)$, as expected, whereas that 'between-genders' was not $(p=0.136)$.

Since several of the biomolecules identified from this RFs analysis had $>1$ discriminatory ISB variable, in such cases only ISBs with the highest MDA values for that biomolecule were retained in order to explore the time dependency of the variables. Therefore, the top 15 ISB features were reduced to 12 key biomarkers (or combinations of them in cases where ISBs contained resonance contributions from 2 such metabolites). Plots of mean ( \pm SEM) normalised ISB intensity values vs. collection time-point for those 12 discriminatory metabolites selected by the RFs model are shown in Figure 4. Lower ISB intensity values for valine, glutamate, hypotaurine/methionine, threonine, tyrosine, lysine/ornithine and phenylalanine were observed at the week 3 time-point than those at subsequent ones for the NP-C1 disease classification, and these observations appear to be consistent with the pre-symptomatic nature of this time-point, i.e. elevated levels of these biomolecules appear 
at the early ( 6 week) and late ( $\geq 9$ week) symptomatic time-points. However, there appeared to be no significant time-dependence of hepatic 3-hydroxyphenylacetate and PEP levels. It is clear from these plots that there were elevated concentrations of valine, glutamate, hypotaurine/methionine, lysine/ornithine, threonine, tyrosine and phenylalanine for the NP-C1 disease classification over those of the combined HET/WT group at all the post-symptomatic time-points, together with lower ones for 3-hydroxyphenylacetate (with the exception of the week 6 time-point samples for this metabolite) and the combined nicotinate/niacinamide variable in this group. Although the admixed nicotinate/niacinamide ISB intensity decreased with increasing time-point for the NP-C1 disease group, such differences were not statistically significant. For the NP-C1 disease classification, the valine, glutamate, tyrosine, phenylalanine, hypotaurine/methionine, lysine/ornithine and threonine predictor biomarker variables all reach maximal levels at week 11.

The univariate ANCOVA model revealed a significant time-point-dependency of some metabolites, particularly phenylalanine $(p=0.019)$, tyrosine $\left(p=2.47 \times 10^{-3}\right)$, valine $(p=3.28$ $\left.\times 10^{-4}\right)$, 3-hydroxyphenylacetate $\left(p=2.24 \times 10^{-3}\right)$, threonine $\left(p=4.56 \times 10^{-4}\right)$ and lysine/ornithine $\left(p=3.17 \times 10^{-4}\right)$. Additionally, this ANCOVA strategy also provided significant $p$ values for the 'between-gender' factor for phenylalanine $(p=0.019)$, tyrosine ( $p$ $=0.014)$, valine $(p=0.028)$ and threonine $(p=0.015)$; these four metabolites showed marginally higher levels for male mice. The combined 'time-point $x$ disease classification' interaction effect was significant for hypotaurine/methionine $(p=0.017)$ and inosine $(p=$ 0.046), and that for the combined 'gender $x$ disease classification' effect was significant for phenylalanine $(p=0.040)$, tyrosine $(p=0.036)$, 3-hydroxyphenylacetate $(p=0.014)$ and 
lysine/ornithine $(p=0.047)$. However, these $p$ values were uncorrected for false discovery rate (FDR).

Performance of the above RFs model, but excluding the pre-symptomatic week 3 samples, gave rise to a slight improvement compared to that achieved without removal, i.e. a mean $\mathrm{OOB}$ error value of $0.16 \pm 1.47 \times 10^{-4}$, and prediction performance values for accuracy, sensitivity and specificity of $0.84 \pm 7.03 \times 10^{-4}, 0.84 \pm 8.76 \times 10^{-4}$ and $0.88 \pm 2.82 \times 10^{-3}$ respectively. Additionally, SEM values estimated for all of these parameters were found to be lower in this improved model; these results are consistent with the pre-symptomatic nature of samples collected at the week 3 time-point in NP-C1 mice.

3.6 ROC analysis: In order to evaluate the ability of our RFs model to classify and predict disease classification status using these ${ }^{1} \mathrm{H}$ NMR ISB biomarker variables, a ROC analysis was performed. With the 12 key biomarker variables selected above (attributable to phenylalanine, tyrosine, valine, methionine/hypotaurine, niacinamide/nicotinate, lysine/ornithine, threonine, glutamate, PEP, inosine, 3-hydroxyphenylacetate and the unassigned $0.77-0.80 \mathrm{ppm}$ ISB), this RFs approach gave a maximum AUROC value of 0.941 (95\% $\mathrm{Cl}=0.823-0.987)$, and predictive accuracies of up to $85.5 \%$ [Figure $5(\mathrm{~b})$ ]. For this purpose, the entire dataset was permuted to generate different RFs models containing increasing numbers of variables from those available, and total numbers of $2,3,5,7,10$ and 12 predictor variables were employed for constructing them. The employment of 12 variables in total was found to provide a highly effective level of discrimination between the two classification groups explored [Figure 5(a)]. 
Key discriminatory biomarker variables featured in the NP-C1 vs. HET comparison were phenylalanine $>$ GSSG $>$ valine $>$ tyrosine $>$ lysine/ornithine $>$ PEP $>$ creatine $>$ hypotaurine/ methionine $>$ unassigned $0.77-0.80 \mathrm{ppm}$ resonance $>$ threonine, whereas those for the NPC1 vs. WT comparison were tyrosine $>$ phenylalanine $>$ hypotaurine $/$ methionine $>$ valine $>$ threonine $>$ glutamate $>$ lysine/ornithine $>$ GSSG > creatine .

3.7 PCA and PCA loadings of the 12 selected biomarker variables: PCA was also applied to this 12 variable dataset [Figure 6(a)], and results acquired clearly confirm a high level of distinction between these two disease group classifications when using this set of biomarkers. Interestingly, the loadings of these 12 variables revealed a cluster containing the majority of the amino acid biomarkers, with higher positive loadings for PC1 [PC1 loadings ranged from 0.27 (phenylalanine) to 0.43 (valine)] and negative ones for PC2. The 0.77-0.80 ppm bucket has PC1 and PC2 loading values very similar to those of PEP, i.e., high loadings for PC2 and intermediate ones for PC1 [Figure 6(b)]. Inosine, together with the 3hydroxyphenylacetate and combined nicotinate/niacinamide signal had relatively high PC2 and low or negative PC1 loadings; a repeat PC loadings analysis also containing individual, isolated nicotinate and niacinamide signals ( $\delta=\mathrm{ppm}$ respectively) demonstrated that both these metabolites had very similar PC1 and PC2 loadings to the combined nicotinate/niacinamide one employed in this model). However, hepatic threonine concentrations appear to be unrelated to the above clusters. Contributions towards higher PCs (PC3, PC4, etc.) were negligible in this model.

Similarly, PLS-DA was employed for the analysis of this dataset containing 12 predictor variables, and both $Q^{2}$ and predictive accuracy values obtained ( 0.62 and 0.92 respectively) 
were the highest for a model system incorporating four components (components 1-4); these quality measures were not significantly improved by the inclusion of one or more of components containing further metabolic predictor variables.

\subsection{Glutathione (GSH/GSSG) redox status of the NP-C1 and HET/WT classifications:}

Although not featuring as significant biomolecule variables in the RFs and further MV analysis models developed, we also explored differences between the mean hepatic concentrations of glutathione (GSH) and its corresponding disulphide oxidation product (GSSG), and in particular that of the [GSH]:[GSSG] concentration ratio, between the NP-C1 and HET/WT classifications investigated. Primarily, it was found that hepatic GSH levels were significantly reduced in NP-C1 mice $(p=0.019)$, although no such differences were noted for GSSG (Figure S3). However, there was a much more highly significant decrease in the mean [GSH]:[GSSG] ratio of the NP-C1 group when compared to that of the HET/WT control one $(p=0.0076)$.

3.9 Metabolic Pathway Analysis: Quantitative mouse pathway topological analysis revealed significant NP-C1 disease-mediated imbalances in tyrosine biosynthesis and metabolism, phenylalanine metabolism, taurine and hypotaurine metabolism, arginine and proline metabolism, nicotinate and niacinamide metabolism, and glycolysis or gluconeogenesis (specifically reaction steps involving PEP), in addition to perturbations in those featuring glutathione and purine (downregulated inosine) metabolism, as listed in Table 3. 
Disturbances to the metabolism of at least some of the above amino acids appear to be common to experimental models of liver fibrosis, ${ }^{33,34}$ and the livers of mutant NP-C1 disease mice become progressively fibrotic in the mouse model employed here ${ }^{35}$ (discussed in section 4.2 below).

\section{DISCUSSION}

4.1 Multicomponent ${ }^{1} \mathrm{H}$ NMR analysis of mouse liver samples: The ${ }^{1} \mathrm{H}$ NMR profiles of mouse liver samples acquired here contained a wide range of metabolites arising from a series of hepatic metabolic processes. Many of the assignments made in the spectra acquired were routinely supported and confirmed via the application of 2D NMR bioanalytical strategies, in particular the ${ }^{1} \mathrm{H}-{ }^{13} \mathrm{C} \mathrm{HSQC}$ and ${ }^{1} \mathrm{H}-{ }^{1} \mathrm{H}$ COSY techniques. In this manner, we were able to confirm the identities and quantitate a total of more than 40 hepatic biomolecules (Table S1).

Preliminary analyses revealed no significant differences in the ${ }^{1} H$ NMR profiles of liver samples collected from WT and HET mice. In addition, no time-dependent or gender-specific variance in hepatic metabolite levels was observed, and in view of this, the HET and WT groups were combined and further MV analysis was focused on comparisons of the combined HET/WT group profiles to those of NP-C1 disease mice. The discriminatory variables identified by RFs analysis were selected amino acids, 3-hydroxyphenylacetate, niacinamide and nicotinate, PEP and inosine, together with an unassigned ISB $(\delta=0.77-0.80$ ppm); the advantages of this approach are detailed in supplementary section S1. 
4.2 Relevance to liver fibrosis-cirrhosis development: Livers from NP-C1 mice exhibit an elevated level of fibrosis, together with hepatocyte apoptosis, inflammation, the infiltration of foamy macrophages, and proliferation of hepatic stellate cells. ${ }^{8,9,36}$ Of these processes, fibrosis plays a predominant role, and is characterised by the replacement of liver tissue by fibrous scar tissue, in addition to regenerative nodules (cirrhosis, which is responsible for major alterations to liver tissue, represents the irreversible end-stage of these developments). Hence, fibrosis gives rise to a progressive loss of liver function, and to a modified liver metabolism. ${ }^{37-40}$ Taken together, collagen and hydroxyproline levels serve as valuable hallmarks for liver fibrosis, the latter as an index of collagen metabolism. ${ }^{41}$

Currently, reports on biochemical and metabolomics investigations of the development of liver fibrosis, and subsequently cirrhosis, in animal models and humans $\left({ }^{1} \mathrm{H}\right.$ NMR-based or otherwise) are limited. However, during the induction of fibrosis-cirrhosis with thioacetamide (TAA), Constantinou et al. $^{33}$ found significant elevations in the hepatic levels of glutamate/glutamine, proline and choline, observations coupled with corresponding decreases in those of acetate, fumarate, TMA, glycogen and inosine. Moreover, in a similar TAA-based animal model, Waters et al. ${ }^{34}$ reported significant elevations in the liver contents of the amino acids isoleucine, leucine, methionine, histidine, phenylalanine and tyrosine, together with increases in those of inosine, adenine, uracil, formate, NMN, creatine, GSH, choline/phosphocholine, and lipids in general; these observations were accompanied by corresponding decreases in TMAO, betaine, glycogen, glucose and niacinamide concentrations. Hence, results from these studies are consistent with the upregulations of hepatic phenylalanine, tyrosine, glutamate and methionine, and downregulations in niacinamide observed in this study. Although the significantly diminished inosine levels 
observed in our NP-C1 disease group are in accordance with the findings $\mathrm{in}^{33}$, this observation contrasts with that found $\mathrm{in}^{34}$.

However, in a HR-MAS NMR investigation of human liver tissue samples collected by needle biopsy performed by Martinez-Granados et al. $^{42}$ found upregulations of glutamate, phosphocholine and phosphoethanolamine, coupled with lower concentrations of choline, TMAO, glucose, glutamine, aspartate and unsaturated fatty acids in cirrhotic livers when expressed relative to those of non-cirrhotic ones, although it should be noted that such differences were only weakly significant in a univariate context (i.e., the $p$ values provided were not corrected for multiple comparisons). Interestingly, glutamate was also featured as a top-15 variable by our RFs MVA, results revealing a significant increase in its hepatic level ( $p=0.0010$ from the ANCOVA model applied).

Moreover, ${ }^{1} \mathrm{H}$ NMR-detectable hydroxyproline was not found to represent a significant hepatic biomarker for the NP-C1 disease group in this study, and nor was it in any of the above fibrosis/cirrhosis-focused NMR investigations.

In a further study, Rajendraa et al. ${ }^{43}$ explored modifications to the hepatic concentrations of free amino acids induced by repeated administration of hexachlorophene (HCP) to male adult mice; this agent exerts pathological modifications to liver structure and morphology (particularly enlargement of bile canaliculi, increases in gap junction numbers, and a disorganisation of tight junctions, together with mitochondrial lesions). ${ }^{44}$ These researchers found that phenylalanine, tyrosine, leucine, isoleucine, valine, threonine, asparagine, glutamate and glutamine levels were significantly elevated following HCP intoxication, whereas that of aspartate was markedly decreased (alanine, glycine, serine and taurine concentrations were unaffected). Moreover, reductions in the specific activity patterns of 
enzymes involved in amino acid metabolism, i.e. those featuring aminotransferases and glutamate dehydrogenase, indicated a suppression of liver amino acid oxidation. The abnormal upregulations in branched chain and aromatic amino acids observed were ascribed to a reduced catabolism in view of a depleted skeletal muscle mass, and hepatic dysfunction respectively; these phenomena may also account for the upregulated liver valine and phenylalanine/tyrosine concentrations observed in this study.

Alam et. al. ${ }^{45}$ monitored a series of inflammatory and fibrosis biomarkers in the livers of

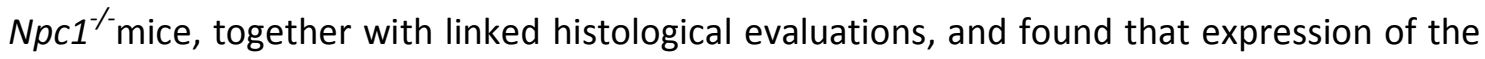
inflammatory genes Cd68 and Itgax (markers of macrophage, and activated macrophage, granulocyte and dendritic cells respectively) were upregulated ca. 88- and 400-fold, respectively, at the late (i.e. $70-83$ days post-induction) disease stages. Furthermore, the Colal (procollagen type a1) marker, which is known to be upregulated during liver fibrosis ${ }^{46}$, was elevated 1.3 -fold in $\mathrm{Npc1}^{\%}$ mice. Additionally, a large number of large foamy macrophages with high cathepsin S precursor (CTSS) levels were found in the livers of 80 day aged $\mathrm{NpCl}^{-/}$mice (unlike that of $\mathrm{NpC}^{-\%}$ mice), together with significant clusters of neutrophils.

More recently, Alam et al. ${ }^{47}$ have reported that the $N p c 1^{n m f 164}$ BALB/C mouse strain, which carries an aspartate-to-glycine mutation at position 105 (D1005G) in the Npc1 gene, displayed markedly elevated plasma levels of liver damage markers, specifically alanine and aspartate aminotransferases (ALT and AST respectively), along with much reduced total protein and albumin concentrations during the 100-day course of this model trial. These observations are, of course, consistent with liver fibrosis-cirrhosis development ${ }^{48}$. 
A full summary of the biomarker status of the significant predictor metabolite variables detected in this investigation in fibrosis-cirrhosis development ${ }^{33-35}$, and also further liver disease/hepatic toxicity ${ }^{43}$, investigations reported is shown in Table 4. These results suggest that the lysine, ornithine, 3-hydroxyphenylacetate, and perhaps also PEP and nicotinate biomarkers discovered here may arise from the pathogenesis of NP-C1 disease rather than fibrosis-cirrhosis, or disease-unspecific liver failure. Therefore, further investigations are required to explore this.

Moreover, a Venn diagram which depicts the similarities and differences in biomarker candidates found for NP-C1 disease mouse model liver, human and mouse model blood plasma/serum, and human urinary biomarkers between this study and those previously reported to date is displayed in Figure 7. This diagram reveals that upregulated valine and nicotinate levels were common to both NP-C1 mouse model system liver (this study) and human urine samples ${ }^{14}$, whereas previous investigations ${ }^{49}$ have detected elevated concentrations of monohexosyl ceramides in both mouse model liver and human blood plasma/serum for this disease. However, although sphingoid bases were found to be upregulated in NP-C1 mouse model liver samples, these levels were significantly diminished in human blood plasma ${ }^{49}$; indeed, there may be a significant inverse correlation between the blood plasma and liver levels of this biomarker in NP-C1 mice and/or humans.

4.3 Upregulations of hepatic amino acids in NP-C1 mice: The results arising from the RFs and further MV statistical analyses conducted revealed that there were clear NP-C1mediated elevations in the concentrations of phenylalanine, tyrosine, lysine/orthinine, hypotaurine/methionine, glutamate, valine and threonine in NP-C1 disease mice. As noted above, these results are generally consistent with the time-dependent manifestation of liver 
fibrosis in this animal model. Such metabolic modifications may be ascribable to muscle proteolysis, in addition to liver parenchyma necrosis. ${ }^{50}$ Moreover, imbalances in hepatic protein biosynthesis and necrosis-induced protein deterioration would also give rise to elevated free amino acid levels in both liver and blood plasma.

The metabolic pathways of phenylalanine and tyrosine are linked through the action of phenylalanine hydroxylase, and the metabolism of both these aromatic amino acids (AAAs) occurs predominantly in the liver. ${ }^{51}$ Elevated blood plasma levels of phenylalanine, threonine, tyrosine, ornithine and methionine have been previously shown to serve key indicators of liver dysfunction and damage. ${ }^{52,53}$. Indeed, Fischer's ratio [the molar ratio of branched-chain amino acids (BCAAs) to those of AAAs (specifically phenylalanine and tyrosine)] in blood plasma has been found to be of much value for evaluating liver metabolism, its dysfunctional severity and hepatic functional reserve. This ratio has long been employed for the analysis of free amino acid concentrations in liver diseases, and diminishes with hepatic damage severity. ${ }^{54}$ These modifications to plasma amino acid concentrations reportedly arise from an enhanced level of BCAA catabolism in muscle coupled with diminished AAA degradation in the failing liver. ${ }^{55}$ However, it should also be noted that plasma BCAA concentrations are critically dependent on nutritional factors, whereas it appears that corresponding AAA levels are determined by intact hepatic metabolism to a much greater extent. In addition, ${ }^{1} \mathrm{H}$ NMR-based metabolomics studies have previously identified increased plasma amino acid levels in rats exposed to a hepatotoxin, $^{56}$ and the detection of modifications to these plasma biomolecule concentrations in patients with liver diseases by this technique have been well documented, and are ascribable to impaired hepatic function, the portal-systemic shunting of blood, and 
hyperinsulinaemia and hyperglucagonaemia. ${ }^{57}$ Hence, it appears that imbalances in liver amino acid contents observed here are linked to NP-C1-associated hepatic damage, including fibrosis. Pathway topological analysis of the key biomarker dataset confirmed the critical importance of disturbances in tyrosine biosynthesis, together with hepatic phenylalanine and nicotinate/niacinamide metabolism in NP-C1 disease (Table 3).

In humans, L-valine degradation, together with that of branched chain amino acids (BCAAs) in general, have been previously shown to be disturbed in two related studies focused on NP-C1 disease: firstly, in an ${ }^{1} \mathrm{H}$ NMR metabolomics urinalysis study, ${ }^{14}$ and secondly in a microarray expression analysis of serum biomarkers. ${ }^{16}$ The upregulated hepatic valine levels found NP-C1 disease mice here, however, may be consistent with those of Ishigure et al., ${ }^{58}$ who found that human liver disease (specifically cirrhosis or hepatocellular carcinoma), diminishes the activities of methacrylyl-CoA hydratase and $\beta$-hydroxyisobutyryl-CoA hydrolase (enzymes involved in valine catabolism), and that decreased levels of these hepatic enzyme activities are derived from post-transcriptional regulation in the damaged liver.

Increases in the plasma concentrations of methionine, together with additional amino acids (including alanine, glutamine and lysine) have also been observed in cases of hepatic failure, ${ }^{59}$ and our results indicate that hepatic methionine levels are also elevated in the mutant NP-C1 disease mouse model investigated. However, since the methionine-C4-C $\underline{H}_{2}$ biomarker ISB signal observed here $(\delta=2.62-2.67 \mathrm{ppm})$ overlapped with the more intense hypotaurine- $\mathrm{CH}_{2} \mathrm{SO}_{2}^{-}$resonance, additional experiments are required to explore this. Previous investigations have revealed that dietary agents containing methionine are 27 
required for the maintenance of normal liver function. ${ }^{60}$ Therefore, any perturbations to methionine metabolism in our mouse model may indeed reflect NP-C1 disease-associated liver dysfunction, and hence there is a requirement for further investigations focused on this amino acid.

4.4 Perturbations to nicotinate and niacinamide metabolites: Both niacinamide and nicotinate are major precursors of $\mathrm{NAD}^{+}$and $\mathrm{NADP}^{+}$synthesis in mammals, although $\mathrm{NAD}^{+}$ can also be synthesized from amino acid precursors, most notably tryptophan (de novo pathway).

In this NP-C1 disease mutant mouse investigation, a decrease in the hepatic ${ }^{1} \mathrm{H}$ NMR ISB intensity of the 8.23-8.29 ppm ISB assigned to both nicotinate and niacinamide-C4-CH functions was observed. Indeed, a fold-change of -1.28 was noted for this ISB, and we have previously shown that metabolites in this pathway are downregulated in the urinary ${ }^{1} \mathrm{H}$ NMR profiles of NP-C1 patients. ${ }^{14}$ In view of their roles as NAD ${ }^{+} / \mathrm{NADH}$ and NADP ${ }^{+} / \mathrm{NADPH}$ precursors, which are employed in a wide range of metabolic redox processes, these observations may be linked to highly significant biochemical and hepatocellular effects. For example, cytochrome P450 activity featured in microsomal oxidation systems are critically dependent on the NADP ${ }^{+} / \mathrm{NADPH}$ coenzyme system. Similarly, the NADP ${ }^{+} / \mathrm{NADPH}$ system is involved in the phenylalanine hydroxylase-mediated oxidative transformation of phenylalanine to tyrosine. A further example from BCAA catabolism involves the oxidative, irreversible decarboxylation of substrates to form branched-chain acyl-CoA adducts (a reaction catalysed by mitochondrial branched-chain $\alpha$-keto acid dehydrogenase), which features the $\mathrm{NAD}^{+} / \mathrm{NADH}$ cofactor system. ${ }^{61}$ 
Also particularly noteworthy is the diminished mean hepatic concentration of GSH present in the NP-C1 mouse classification (section 4.6). These results may reflect a disease-mediated imbalance in hepatocyte [NADP $\left.{ }^{+}\right]:[\mathrm{NADPH}]$ concentration ratios, since GSH is required for the reconversion of $\mathrm{NADP}^{+}$to $\mathrm{NADPH}^{62}$

Additionally, disruption of the $\mathrm{NAD}^{+} / \mathrm{NADP}$ cycle may be linked to the dysregulation of lysosomal calcium previously observed In NP-C1 disease. ${ }^{63}$ This lysosomal release of $\mathrm{Ca}^{2+}$ arises from the actions of the $\mathrm{Ca}^{2+}$-mobilising second messenger nicotinic acid adenine dinucleotide phosphate (NAADP). ${ }^{63}$ Although mechanisms for the biosynthesis of NAADP remain speculative, one probable route is the base-exchange process involving reaction of NADP with nicotinate to generate both NAADP and niacinamide, which is catalysed by ADPribosyl cyclases. ${ }^{64}$ Since both nicotinate and niacinamide were ${ }^{1} \mathrm{H}$ NMR-detectable in this study, and both were represented as a significantly downregulated admixed ISB predictor variable in the NP-C1 disease classification, we tested for any differences in the hepatic molar concentration ratios of niacinamide:nicotinate between the NP-C1 and HET/WT classifications. Although this ratio was $c a .47 \%$ greater in the NP-C1 group, this difference was found not to be statistically significant $(p=0.082)$.

Consistent with our observations, Waters et al. ${ }^{34}$ also found a downregulation of hepatic niacinamide in their ${ }^{1} \mathrm{H}$ NMR-linked metabolomics study of thioacetamide-driven fibrosis/cirrhosis in experimental animals, and this was ascribed to an enhanced level of this metabolite's methylation to 1-methylnicotinamide in cirrhotic liver. Furthermore, VarelaRey et al. ${ }^{65}$ found that orally-administered niacinamide ameliorated fatty liver and fibrosis in 
glycine $\mathrm{N}$-methyltransferase knockout mice, and concluded that this agent acted through the prevention of hepatic fat accumulation and apoptosis via reductions in liver Sadenosylmethionine content.

In view of the potential far-reaching implications of any disturbances to the $\mathrm{NAD}^{+} / \mathrm{NADP}^{+}$ cycles, and also to NAADP biosynthesis, it is possible that the statistically significant differences in liver metabolite concentrations observed here could be traced back to defects in metabolic pathways linked to the early-onset, rapidly progressive form of the clinical NPC1 phenotype, ${ }^{66,67}$ which is associated with hepatic dysfunction, together with infantile psychomotor delay. ${ }^{68}$

\subsection{3-Hydroxyphenylacetate as a potential biomarker for NP-C1 disease-associated liver}

dysfunction: Intriguingly, the ISB intensity of 3-hydroxyphenylacetate was found to be significantly lower in the NP-C1 group than that of the control HET/WT one. Moreover, it was found to be strongly associated with a PC also featuring nicotinate and niacinamide, together with PEP and the unassigned $0.77-0.80$ ppm ISB [Figure 6(b)]. 3hydroxyphenylacetate is involved in the human tyrosine metabolism pathway, in which it is transformed to 3,4-dihydroxyphenylacetate via the enzyme 4-hydroxyphenylacetate 3mono-oxygenase. This reaction also requires $\mathrm{NAD}^{+} / \mathrm{NADH}$ as a coenzyme system (equation 2 ), and which is synthesized through the nicotinate/niacinamide pathway, metabolites of which were also identified as discriminatory variables here.

3-hydroxyphenylacetate $+\mathrm{O}_{2}+\mathrm{NADH}+\mathrm{H}^{+} \leftrightarrow$ 3,4-dihydroxyphenylacetate $+\mathrm{NAD}^{+}+\mathrm{H}_{2} \mathrm{O}$ 
Imbalances in 3-hydroxyphenylacetate biosynthesis may indicate that perturbations in the hepatic $\left[\mathrm{NAD}^{+}\right]:[\mathrm{NADH}]$ molar concentration ratio (an index of mitochondrial redox status) in NP-C1 disease mice, a process favouring oxidative reaction processes in vivo, as noted above (section 4.4). Hence, disturbances in the metabolism of 3,4-dihydroxyphenylacetete to 3hydroxyphenylacetate (suppressing production of the latter) in mutant NP-C1 disease mouse liver tissue may serve to partially conserve oxidising $\mathrm{NAD}^{+}$equivalents required for further metabolic processes, possibly one or more of those required for the upregulation of metabolites identified as biomarkers here.

However, the potential applications of 3-hydroxyphenylacetate as a biomarker for NP-C1 disease-associated liver disease are complicated, since this phenolic acid anion is also generated as a co-metabolite arising from gut microflora. ${ }^{69}$ Indeed, this agent appears to serve as a valuable marker for bacterial dysbiosis, and has been demonstrated to appear when bacteria are introduced to germ-free rats. ${ }^{70}$ Therefore, imbalances in the activity of such microflora in mutant NP-C1 disease mice may also account for the significantly lower hepatic levels of this metabolite found in this group. Indeed, in a recent study, we found that human urinary concentrations of the co-metabolite trimethylamine were significantly elevated ( $>2$-fold) over those of a heterozygous carrier control group, an observation also reflecting significant NP-C1 disease-induced modifications to the metabolic activities of gut microflora. Moreover, Mardinoglu et al. ${ }^{71}$ found that gut microbiota modulates host amino acids and GSH metabolism in mice, observations also relevant to our findings here.

A further complication is that 3-hydroxyphenylacetate may also arise directly from dietary sources $^{72}$, and it may be generated from dietary polyphenols which are resistant to the 31 
actions of digestive enzymes. Notably, urinary levels of this agent are substantially increased when humans are fed catechin and chocolate (the latter being a rich source of proanthocyanidin), ${ }^{73}$ and previous investigations employing a human anaerobic fecal fermentation model revealed that quercetin was metabolised to 3,4dihydroxyphenylacetate within $2 \mathrm{hr}$., and that the latter metabolite was then transformed to 3 -hydroxyphenylacetate during a subsequent $8 \mathrm{hr}$. period. ${ }^{74}$ Hence, these additional factors represent further confounding limitations regarding the use of this agent as a potential biomarker. Moreover, this metabolite serves as an effective antioxidant, and hence its lower hepatic levels observed here conceivably also arise from its oxidative consumption by reactive oxygen species (ROS) such as hydroxyl radical ( $\left.{ }^{\circ} \mathrm{OH}\right)$.

Previous reports on the value of 3-hydroxyphenylacetate as a biomarker are somewhat limited, but in one study Boudonck et. al. $^{75}$ found that this metabolite, together with hippurate, served as a progressive downregulated urinary marker of nephrotoxicity in rats treated with three distinct nephrotoxins. Indeed, 3-hydroxyphenylacetate concentrations were reduced up to 7-fold in these samples, and the researchers involved concluded that this biomolecule featured as a very early and valuable biomarker of nephrotoxicity. Moreover, abnormal urinary levels of this metabolite have also been reported in both eosinophilic esophagitis and gastrointestinal reflux disease ${ }^{76}$.

4.6 Metabolism and potential antioxidant (ROS-scavenging) roles of hypotaurine: The role of hypotaurine as a potential biomarker for NP-C1 disease-associated liver dysfunction is complicated in view of the partial overlap of its $\mathrm{C}_{4}-\underline{\mathrm{C}}_{2} \mathrm{SO}_{2}{ }^{-}$resonance with that of methionine's- $\mathrm{C} 4-\mathrm{CH}_{2}$ one, as noted above. However, if its role as a biomarker here is 
confirmed, hypotaurine serves as a key antioxidant defence in mitochondria and further intracellular environments, and is oxidised by ROS to yield taurine, which is subsequently excreted through its transporter into extracellular media. ${ }^{77}$ The production of ROS has been indicated as one of the pathogenic effects arising from NP-C1 disease pathogenesis, and consistent with this hypothesis, elevated levels of cholesterol oxidation products have been reported in the blood plasma of NP-C1 disease patients, and also in the liver of NP-C1 mutant mice. ${ }^{78}$ In view of the involvement of oxidative stress in this disease process, ${ }^{79}$ the increased hepatic generation of hypotaurine noted here may arise as an essential metabolic response stimulated to combat against it. ${ }^{80}$ The diminished GSH:GSSG molar ratios observed in NP-C1 mouse livers are also consistent with an elevated level of hepatic oxidative stress in this animal model (section 4.7).

Several previous studies performed in NP-C1-deficient mice indicate that hepatocyte apoptosis may serve as a primary cause of progressive liver degeneration and failure in this animal model, ${ }^{8,36}$ and/or an increased activation of macrophages ${ }^{10}$; such considerations may also be applicable to human NP-C1 patients. If confirmed, higher NP-C1 hepatic hypotaurine levels observed may be consistent with an enhanced metabolism of cysteine by the hepatic cysteine dioxygenase/cysteine sulphinate decarboxylase pathway stimulated by an upregulation of oxidative stress episodes, which serve as key mediators of hepatocyte apoptosis. However, with regard to macrophage activation, we found no evidence for NP-C1 disease-mediated increases in levels of nitric oxide (NO')-producing arginine in the NP-C1 mutant classification liver samples. 
4.7 Imbalances in hepatic GSH/GSSG redox status in NP-C1 disease mice: The reduced hepatic [GSH]:[GSSG] molar ratios observed in the NP-C1 mouse model are consistent with the development of liver fibrosis as a pathological feature. Indeed, it is well known that tissue GSH concentrations significantly diminish in both experimental fibrosis models and in human fibrotic diseases, although the precise mechanism and biological significance of these depletions remain unclear. ${ }^{81}$ Although there is also much evidence available indicating that such changes in hepatic GSH levels arise from an increased level of ROS-mediated oxidative stress ${ }^{82}$ (which itself appears to play an important role in the development of fibrotic diseases in humans), alternative hypotheses are that it may also be ascribable to a dysregulation of the synthesis of this small molecule antioxidant during liver injury/fibrosis, or more simply the availability of cysteine, which may be limited in view of nutritional deficiencies and/or multiple perturbations to the methionine metabolic pathway. ${ }^{83}$ Hence, results from these studies are consistent with the upregulations of hepatic phenylalanine, threonine, tyrosine, methionine and valine observed in this study.

4.8 Perturbations to hepatic PEP concentrations in NP-C1 disease mice: PEP is generated via the actions of the enzyme enolase on 2-phosphoglycerate, and subsequently it is metabolised to pyruvate by pyruvate kinase, a process yielding ATP through substrate level phosphorylation (these represent the 9th and 10th step of the glycolysis pathway, respectively) $)^{84}$. However, in gluconeogenesis, PEP is produced from oxaloacetate decarboxylation, a process which involves the hydrolysis of guanosine triphosphate (GTP) ${ }^{84}$. This reaction is the rate-limiting stage of this process, and is catalysed by PEP carboxykinase (PEPCK). Therefore, disturbances to either of these pathways (specifically, malfunctions in the above enzymes, and particularly that involved in the rate-determining step of 
gluconeogenesis) may indeed have some profound consequences regarding hepatic function and integrity in this NP-C1 disease mouse model.

4.9 Inosine as a potential biomarker feature: Inosine is hepatically-metabolised, and arises from the transformation of adenine to adenosine or inosine monophosphate (IMP), each of which may then be converted to inosine ${ }^{84}$. Moreover, the enzyme purine nucleoside phosphorylase intra-converts inosine and hypoxanthine. Notably, inosine has not been previously reported as a biomarker for fibrosis-cirrhosis, nor non-specific liver dysfunction or failure, and hence its diminished hepatic concentrations may arise as a consequence of NP-C1 disease pathology. Moreover, inosine also serves as an essential intermediate in a chain of purine nucleotide reactions which are involved in muscle movement. In a mouse model of septic shock, intraperitoneally-administered inosine has been found to alleviate systemic inflammation, organ damage, vascular dysfunction and tissue hypoxia ${ }^{85}$. Indeed, in these studies, inosine administration was also found to elevate the hepatic [NAD $]$ :[NADH] ratio, which serves as an index of mitochondrial redox status ${ }^{86}$. Therefore, the lower hepatic levels of inosine detectable in NP-C1 mice may again be consistent with imbalances in this ratio.

\section{CONCLUSIONS}

${ }^{1} \mathrm{H}$ NMR-linked metabolomics techniques provide much valuable biomolecular information reflecting hepatic dysfunction or damage in the NP-C1 disease mouse model system, including the development of fibrosis. Although many of our observations are generally consistent with the development of fibrosis-cirrhosis (or alternative liver dysfunctions) in 
NP-C1 mice (i.e. increased hepatic levels of selected amino acids such as AAAs and glutamate, together with diminished GSH and niacinamide concentrations), it appears that upregulated lysine/ornithine, and downregulated 3-hydroxyphenylacetate, PEP and inosine, are not. Contingent disturbances to the ${ }^{1} \mathrm{H}$ NMR metabolic profiles of human biofluids (e.g., blood plasma or urine) could, at least in principle, be employed to monitor the aetiology, pathogenesis and severity of liver damage in NP-C1 patients. Our observations are consistent with modifications to the blood plasma amino acid patterns of both humans and experimental animals which are induced by chronic liver failure, specifically elevated concentrations of tyrosine and phenylalanine, in addition to that of methionine. Downregulation in the hepatic nicotinate/niacinamide concentrations observed here could be linked to dysfunctions in the $\mathrm{NAD}^{+} / \mathrm{NADH}$ cycle and the biosynthesis of $\mathrm{Ca}^{2+}$-mobilising second messenger NAADP which may, at least in principle, exert a substantial range of physiological effects, and also be associated with further metabolic disturbances observed in NP-C1 disease mice.

\section{Associated Content}

\section{Supplementary Material}

Table S1: ${ }^{1} \mathrm{H}-\mathrm{NMR}$ resonance assignments with chemical shifts, multiplicities, and coupling constant values of signals identified in aqueous liver sample extracts (page S2).

Figure S1. Receiver operating characteristic (ROC) curve exploration and testing probability view for direct comparisons of the NP-C1 disease classification with the (a) WT and (b) HET ones (page S4).

Figure S2: $400 \mathrm{MHz} 2 \mathrm{D}^{1} \mathrm{H}-{ }^{1} \mathrm{H}$ COSY NMR profiles of aqueous liver sample extracts (page S5). 36 
Figure S3: Box plots of TSP-normalised and Pareto-scaled intensities of GSSG and GSH ${ }^{1} \mathrm{H}$ NMR resonances (Table S1), and the GSH:GSSG molar ratio (page S6)

Section S1: Metabolomic advantages offered by the Random Forests (RFs) multivariate analysis strategy employed (page S7).

Figure S4: Mean decrease in accuracy (MDA) values computed for the 5 most effective discriminatory variables throughout 100 iterations (page S8).

\section{Supplementary References (page S8)}

Acknowledgements: We are very grateful to the SPARKS Medical Charity for Children's Health for providing funding for a Research Fellowship to F.P., and the Hope Against Cancer Charity and De Montfort University, Leicester for the provision of doctoral training bursaries to V. R-R. E-R N. is supported by Action Medical research, and DS by the Niemann-Pick Research Foundation. F. M. P. is a Royal Society Wolfson Research Merit Award holder.

\section{REFERENCES}

(1) Vanier, M. T. Niemann-Pick disease type C. Orphanet J. Rare Dis. 2010, 5, 16-1172-5-16.

(2) NP-C Guidelines Working Group; Wraith, J. E.; Baumgartner, M. R.; Bembi, B.; Covanis, A.; Levade, T.; Mengel, E.; Pineda, M.; Sedel, F.; Topcu, M.; Vanier, M. T.; Widner, H.; 
Wijburg, F. A.; Patterson, M. C. Recommendations on the diagnosis and management of Niemann-Pick disease type C. Mol. Genet. Metab. 2009, 98, 152-165.

(3) Vanier, M. T.; Millat, G. Niemann-Pick disease type C. Clin Genet. 2003, 64, 269-281.

(4) Sun, X.; Marks, D. L.; Park, W. D.; Wheatley, C. L.; Puri, V.; O'Brien, J. F.; Kraft, D. L.; Lundquist, P. A.; Patterson, M. C.; Pagano, R. E.; Snow, K. Niemann-Pick C variant detection by altered sphingolipid trafficking and correlation with mutations within a specific domain of NPC1. Am. J. Hum. Genet. 2001, 68, 1361-1372.

(5) Vance, J. E.; Karten, B. Niemann-Pick C disease and mobilization of lysosomal cholesterol by cyclodextrin. J. Lipid Res. 2014, 55, 1609-1621.

(6) Kelly, D. A.; Portmann, B.; Mowat, A. P.; Sherlock, S.; Lake, B. D. Niemann-Pick disease type C: diagnosis and outcome in children, with particular reference to liver disease. J. Pediatr. 1993, 123, 242-247.

(7) Dietschy, J. M.; Turley, S. D. Control of cholesterol turnover in the mouse. J. Biol. Chem. 2002, 277, 3801-3804.

(8) Beltroy, E. P.; Richardson, J. A.; Horton, J. D.; Turley, S. D.; Dietschy, J. M. Cholesterol accumulation and liver cell death in mice with Niemann-Pick type C disease. Hepatology $2005,42,886-893$.

(9) Rimkunas, V. M.; Graham, M. J.; Crooke, R. M.; Liscum, L. In vivo antisense oligonucleotide reduction of NPC1 expression as a novel mouse model for Niemann Pick type C- associated liver disease. Hepatology 2008, 47, 1504-1512. 
(10) Liu, B.; Turley, S. D.; Burns, D. K.; Miller, A. M.; Repa, J. J.; Dietschy, J. M. Reversal of defective lysosomal transport in NPC disease ameliorates liver dysfunction and neurodegeneration in the npc1-/- mouse. Proc. Natl. Acad. Sci. U. S. A. 2009, 106, 23772382.

(11) Owusu-Sarfo, K.; Asiago, V. M.; Deng, L.; Gu, H.; Wei, S.; Shanaiah, N.; Nagana Gowda, G. A.; Xi, B.; Chiorean, E. G.; Raftery, D. NMR-based Metabolite Profiling of Pancreatic Cancer. Curr. Metabolomics 2014, 2, 204-212.

(12) Serkova, N. J.; Jackman, M.; Brown, J. L.; Liu, T.; Hirose, R.; Roberts, J. P.; Maher, J. J.; Niemann, C. U. Metabolic profiling of livers and blood from obese Zucker rats. J. Hepatol. 2006, 44, 956-962.

(13) Whigham, L. D.; Butz, D. E.; Dashti, H.; Tonelli, M.; Johnson, L. K.; Cook, M. E.; Porter, W. P.; Eghbalnia, H. R.; Markley, J. L.; Lindheim, S. R.; Schoeller, D. A.; Abbott, D. H.; AssadiPorter, F. M. Metabolic Evidence of Diminished Lipid Oxidation in Women With Polycystic Ovary Syndrome. Curr. Metabolomics 2014, 2, 269-278.

(14) Ruiz-Rodado, V.; Luque-Baena, R. M.; te Vruchte, D.; Probert, F.; Lachmann, R. H.; Hendriksz, C. J.; Wraith, J. E.; Imrie, J.; Elizondo, D.; Sillence, D.; Clayton, P.; Platt, F.; Grootveld, M. 1H NMR-Linked Urinary Metabolic Profiling of Niemann-Pick Class C1 (NPC1) Disease: Identification of Potential New Biomarkers using Correlated Component Regression (CCR) and Genetic Algorithm (GA) Analysis Strategies. Curr. Metabolomics 2014, 2, 88-121. 
(15) Kennedy, B. E.; LeBlanc, V. G.; Mailman, T. M.; Fice, D.; Burton, I.; Karakach, T. K.; Karten, B. Pre-symptomatic activation of antioxidant responses and alterations in glucose and pyruvate metabolism in Niemann-Pick Type C1-deficient murine brain. PLoS One 2013, 8, e82685.

(16) Cluzeau, C. V.; Watkins-Chow, D. E.; Fu, R.; Borate, B.; Yanjanin, N.; Dail, M. K.; Davidson, C. D.; Walkley, S. U.; Ory, D. S.; Wassif, C. A.; Pavan, W. J.; Porter, F. D. Microarray expression analysis and identification of serum biomarkers for NiemannPick disease, type C1. Hum. Mol. Genet. 2012, 21, 3632-3646.

(17) Vinaixa, M.; Rodriguez, M. A.; Rull, A.; Beltran, R.; Blade, C.; Brezmes, J.; Canellas, N.; Joven, J.; Correig, X. Metabolomic assessment of the effect of dietary cholesterol in the progressive development of fatty liver disease. J. Proteome Res. 2010, 9, 2527-2538.

(18) Loftus, S. K.; Morris, J. A.; Carstea, E. D.; Gu, J. Z.; Cummings, C.; Brown, A.; Ellison, J.; Ohno, K.; Rosenfeld, M. A.; Tagle, D. A.; Pentchev, P. G.; Pavan, W. J. Murine model of Niemann-Pick C disease: mutation in a cholesterol homeostasis gene. Science 1997, $277,232-235$.

(19) Baudry, M.; Yao, Y.; Simmons, D.; Liu, J.; Bi, X. Postnatal development of inflammation in a murine model of Niemann-Pick type C disease: immunohistochemical observations of microglia and astroglia. Exp. Neurol. 2003, 184, 887-903.

(20) Pentchev, P. G.; Boothe, A. D.; Kruth, H. S.; Weintroub, H.; Stivers, J.; Brady, R. O. A genetic storage disorder in BALB/C mice with a metabolic block in esterification of exogenous cholesterol. J. Biol. Chem. 1984, 259, 5784-5791. 
(21) Morris, M. D.; Bhuvaneswaran, C.; Shio, H.; Fowler, S. Lysosome lipid storage disorder in NCTR-BALB/c mice. I. Description of the disease and genetics. Am. J. Pathol. 1982, 108, 140-149.

(22) te Vruchte, D.; Lloyd-Evans, E.; Veldman, R. J.; Neville, D. C.; Dwek, R. A.; Platt, F. M.; van Blitterswijk, W. J.; Sillence, D. J. Accumulation of glycosphingolipids in NiemannPick C disease disrupts endosomal transport. J. Biol. Chem. 2004, 279, 26167-26175.

(23) Waters, N. J.; Holmes, E.; Williams, A.; Waterfield, C. J.; Farrant, R. D.; Nicholson, J. K. NMR and pattern recognition studies on the time-related metabolic effects of alphanaphthylisothiocyanate on liver, urine, and plasma in the rat: an integrative metabonomic approach. Chem. Res. Toxicol. 2001, 14, 1401-1412.

(24) Jiang, C. Y.; Yang, K. M.; Yang, L.; Miao, Z. X.; Wang, Y. H.; Zhu, H. B. A H NMR-Based Metabonomic Investigation of Time-Related Metabolic Trajectories of the Plasma, Urine and Liver Extracts of Hyperlipidemic Hamsters. PLoS One 2013, 8, e66786.

(25) Wishart, D. S.; Jewison, T.; Guo, A. C.; Wilson, M.; Knox, C.; Liu, Y.; Djoumbou, Y.; Mandal, R.; Aziat, F.; Dong, E.; Bouatra, S.; Sinelnikov, I.; Arndt, D.; Xia, J.; Liu, P.; Yallou, F.; Bjorndahl, T.; Perez-Pineiro, R.; Eisner, R.; Allen, F.; Neveu, V.; Greiner, R.; Scalbert, A. HMDB 3.0--The Human Metabolome Database in 2013. Nucleic Acids Res. 2013, 41, D801-7.

(26) Smilde, A. K.; Jansen, J. J.; Hoefsloot, H. C.; Lamers, R. J.; van der Greef, J.; Timmerman, M. E. ANOVA-simultaneous component analysis (ASCA): a new tool for analyzing designed metabolomics data. Bioinformatics 2005, 21, 3043-3048. 
(27) Xia, J.; Sinelnikov, I. V.; Han, B.; Wishart, D. S. MetaboAnalyst 3.0--making metabolomics more meaningful. Nucleic Acids Res. 2015, 43, W251-7.

(28) Liaw, A.; Wiener, M. Classification and regression by randomForest. R. News 2002. 2:1822.

(29) Breiman, L. Random Forest. Mach Learn 2001, 45, 5-32.

(30) Bureau, A.; Dupuis, J.; Hayward, B.; Falls, K.; Van Eerdewegh, P. Mapping complex traits using Random Forests. 2003, 4 Suppl 1, S64.

(31) Xia, J.; Broadhurst, D. I.; Wilson, M.; Wishart, D. S. Translational biomarker discovery in clinical metabolomics: an introductory tutorial. Metabolomics 2013, 9, 280-299.

(32) Aittokallio, T.; Schwikowski, B. Graph-based methods for analysing networks in cell biology. Brief Bioinform 2006, 7, 243-255.

(33) Constantinou, M. A.; Theocharis, S. E.; Mikros, E. Application of metabonomics on an experimental model of fibrosis and cirrhosis induced by thioacetamide in rats. Toxicol. Appl. Pharmacol. 2007, 218, 11-19.

(34) Waters, N. J.; Waterfield, C. J.; Farrant, R. D.; Holmes, E.; Nicholson, J. K. Metabonomic deconvolution of embedded toxicity: application to thioacetamide hepato- and nephrotoxicity. Chem. Res. Toxicol. 2005, 18, 639-654.

(35) Sayre, N. L.; Rimkunas, V. M.; Graham, M. J.; Crooke, R. M.; Liscum, L. Recovery from liver disease in a Niemann-Pick type C mouse model. J. Lipid Res. 2010, 51, 2372-2383. 
(36) Rimkunas, V. M.; Graham, M. J.; Crooke, R. M.; Liscum, L. TNF-\{alpha\} plays a role in hepatocyte apoptosis in Niemann-Pick type C liver disease. J Lipid Res 2009, 50, 327333.

(37) Friedman, S. L. Liver fibrosis -- from bench to bedside. J. Hepatol. 2003, 38 Suppl 1, S3853.

(38) Pinzani, M.; Rombouts, K.; Colagrande, S. Fibrosis in chronic liver diseases: diagnosis and management. J. Hepatol. 2005, 42 Suppl, S22-36.

(39) Dezortova, M.; Taimr, P.; Skoch, A.; Spicak, J.; Hajek, M. Etiology and functional status of liver cirrhosis by 31P MR spectroscopy. World J. Gastroenterol. 2005, 11, 6926-6931.

(40) Kuriyama, S.; Yokoyama, F.; Inoue, H.; Takano, J.; Ogawa, M.; Kita, Y.; Yoshiji, H.; Deguchi, A.; Kimura, Y.; Himoto, T.; Yoneyama, H.; Kurokohchi, K.; Masaki, T.; Uchida, N.; Watanabe, S. Sequential assessment of the intrahepatic expression of epidermal growth factor and transforming growth factor-beta1 in hepatofibrogenesis of a rat cirrhosis model. Int. J. Mol. Med. 2007, 19, 317-324.

(41) Toyoki, Y.; Sasaki, M.; Narumi, S.; Yoshihara, S.; Morita, T.; Konn, M. Semiquantitative evaluation of hepatic fibrosis by measuring tissue hydroxyproline. Hepatogastroenterology 1998, 45, 2261-2264.

(42) Martinez-Granados, B.; Morales, J. M.; Rodrigo, J. M.; Del Olmo, J.; Serra, M. A.; Ferrandez, A.; Celda, B.; Monleon, D. Metabolic profile of chronic liver disease by NMR spectroscopy of human biopsies. Int. J. Mol. Med. 2011, 27, 111-117. 
(43) Rajendra, W.; Prasad, G. V.; Indira, K. Deviations in hepatic amino acid profiles of mouse following repeated hexachlorophene administration. J. Environ. Sci. Health B. 1988, 23, 409-426.

(44) Robenek, H.; Meiss, R.; Themann, H.; Hulsbusch, R. Thin section and freeze-fracture studies of hexachlorophene induced alterations in the rat liver with special regard to intercellular junctions. Exp. Pathol. (Jena) 1980, 18, 257-268.

(45) Alam, Md. S.; Getz, M.; Yi, S.; Kurkewich, J.; Safeukui, I.; Halder, K. Plasma signature of neurological disease in the monogenetic disorder Niemann-Pick Type C. J. Biol. Chem. 2014, 289(12), 8051-8066.

(46) Sayre, NL.; Rimkunas, V. M.; Graham, M. J.; Crooke, R. M.; Liscum, L. Recovery from liver disease in a Niemann-Pick type C mouse model. J. Lipid Res. 2010, 51, 2372-2383.

(47) Alam Md. S.; Getz, M.; Haldar K. Chronic administration of an HDAC inhibitor treats both neurological and systemic Niemann-Pick type $\mathrm{C}$ disease in a mouse model. Science Translational Medicine 2016 Feb. 178 (326), 326ra23. [doi: 10.1126/scitranslmed.aad9407]

(48) Battaller, R.; Brenner, D. A. Liver fibrosis. Journal of Clinical Investigation 2005, 115(2), 209-218.

[49] Fan, M.; Sidhu, R.; Fujiwara, H.; Tortelli, B.; Zhang, J.; Davidson, C.; Walkley, S. U.; Bagel, J. H.; Vite, C.; Yanjanin, N. M.; Porter, F. D.; Schaffer, J. E.; Ory, D. S. Identification of Niemann-Pick C1 disease biomarkers through sphingolipid profiling. J. Lipid Res. 2013, 54(10), 2800-2814. doi: 10.1194/jlr.M040618. Epub 2013 Jul 23. 
(50) Fontana, L.; Moreira, E.; Torres, M. I.; Fernandez, M. I.; Rios, A.; Sanchez de Medina, F.; Gil, A. Serum amino acid changes in rats with thioacetamide-induced liver cirrhosis. Toxicology 1996, 106, 197-206.

(51) Fernstrom, J. D.; Fernstrom, M. H. Tyrosine, phenylalanine, and catecholamine synthesis and function in the brain. J. Nutr. 2007, 137, 1539S-1547S.

(52) Pierre, G.; Chronopoulou, E. Metabolic disorders presenting as liver disease. J Paediatr Child Health 2013, 23, 509-515.

(53) Clayton, P. T. Diagnosis of inherited disorders of liver metabolism. J. Inherit. Metab. Dis. 2003, 26, 135-146.

(54) Ishikawa, T. Branched-chain amino acids to tyrosine ratio value as a potential prognostic factor for hepatocellular carcinoma. World J. Gastroenterol. 2012, 18, 20052008.

(55) Soeters, P. B.; Fischer, J. E. Insulin, glucagon, aminoacid imbalance, and hepatic encephalopathy. Lancet 1976, 2, 880-882.

(56) Yap, I. K.; Clayton, T. A.; Tang, H.; Everett, J. R.; Hanton, G.; Provost, J. P.; Le Net, J. L.; Charuel, C.; Lindon, J. C.; Nicholson, J. K. An integrated metabonomic approach to describe temporal metabolic disregulation induced in the rat by the model hepatotoxin allyl formate. J. Proteome Res. 2006, 5, 2675-2684.

(57) Sunny, N. E.; Kalavalapalli, S.; Bril, F.; Garrett, T. J.; Nautiyal, M.; Mathew, J. T.; Williams, C. M.; Cusi, K. Cross-talk between branched-chain amino acids and hepatic 
mitochondria is compromised in nonalcoholic fatty liver disease. Am. J. Physiol. Endocrinol. Metab. 2015, 309, E311-9.

(58) Ishigure, K.; Shimomura, Y.; Murakami, T.; Kaneko, T.; Takeda, S.; Inoue, S.; Nomoto, S.; Koshikawa, K.; Nonami, T.; Nakao, A. Human liver disease decreases methacrylyl-CoA hydratase and beta-hydroxyisobutyryl-CoA hydrolase activities in valine catabolism. Clin. Chim. Acta 2001, 312, 115-121.

(59) Sato, K.; Fukushima, D.; Doi, H.; Satomi, S. Higher serum methionine levels as a predictive factor in patients with irreversible fulminant hepatic failure. Transplant. Proc. 2013, 45, 1904-1906.

(60) Mato, J. M.; Martinez-Chantar, M. L.; Lu, S. C. Methionine metabolism and liver disease. Annu. Rev. Nutr. 2008, 28, 273-293.

(61) Hutson, S. M.; Sweatt, A. J.; Lanoue, K. F. Branched-chain [corrected] amino acid metabolism: implications for establishing safe intakes. J. Nutr. 2005, 135, 1557S-64S.

(62) Lushchak, V. I. Glutathione homeostasis and functions: potential targets for medical interventions. J. Amino Acids 2012, 2012, 736837.

(63) Lloyd-Evans, E.; Morgan, A. J.; He, X.; Smith, D. A.; Elliot-Smith, E.; Sillence, D. J.; Churchill, G. C.; Schuchman, E. H.; Galione, A.; Platt, F. M. Niemann-Pick disease type C1 is a sphingosine storage disease that causes deregulation of lysosomal calcium. Nat. Med. 2008, 14, 1247-1255. 
(64) Graeff, R.; Liu, Q.; Kriksunov, I. A.; Hao, Q.; Lee, H. C. Acidic residues at the active sites of CD38 and ADP-ribosyl cyclase determine nicotinic acid adenine dinucleotide phosphate (NAADP) synthesis and hydrolysis activities. J. Biol. Chem. 2006, 281, 2895128957.

(65) Varela-Rey, M.; Martinez-Lopez, N.; Fernandez-Ramos, D.; Embade, N.; Calvisi, D. F.; Woodhoo, A.; Rodriguez, J.; Fraga, M. F.; Julve, J.; Rodriguez-Millan, E.; Frades, I.; Torres, L.; Luka, Z.; Wagner, C.; Esteller, M.; Lu, S. C.; Martinez-Chantar, M. L.; Mato, J. M. Fatty liver and fibrosis in glycine $\mathrm{N}$-methyltransferase knockout mice is prevented by nicotinamide. Hepatology 2010, 52, 105-114.

(66) Fink, J. K.; Filling-Katz, M. R.; Sokol, J.; Cogan, D. G.; Pikus, A.; Sonies, B.; Soong, B.; Pentchev, P. G.; Comly, M. E.; Brady, R. O. Clinical spectrum of Niemann-Pick disease type C. Neurology 1989, 39, 1040-1049.

(67) Omura, K.; Suzuki, Y.; Norose, N.; Sato, M.; Maruyama, K.; Koeda, T. Type C NiemannPick disease: clinical and biochemical studies on 6 cases. Brain Dev. 1989, 11, 57-61.

(68) Garver, W. S.; Jelinek, D.; Meaney, F. J.; Flynn, J.; Pettit, K. M.; Shepherd, G.; Heidenreich, R. A.; Vockley, C. M.; Castro, G.; Francis, G. A. The National Niemann-Pick Type C1 Disease Database: correlation of lipid profiles, mutations, and biochemical phenotypes. J. Lipid Res. 2010, 51, 406-415. 
(69) Konishi, Y. Transepithelial transport of microbial metabolites of quercetin in intestinal Caco-2 cell monolayers. J. Agric. Food Chem. 2005, 53, 601-607.

(70) Lord, R. S.; Bralley, J. A. Clinical applications of urinary organic acids. Part 2. Dysbiosis markers. Altern. Med. Rev. 2008, 13, 292-306.

(71) Mardinoglu, A.; Shoaie, S.; Bergentall, M.; Ghaffari, P.; Zhang, C.; Larsson, E.; Backhed, F.; Nielsen, J. The gut microbiota modulates host amino acid and glutathione metabolism in mice. Mol. Syst. Biol. 2015, 11, 834.

(72) Prior, R. L.; Rogers, T. R.; Khanal, R. C.; Wilkes, S. E.; Wu, X.; Howard, L. R. Urinary excretion of phenolic acids in rats fed cranberry. J. Agric. Food Chem. 2010, 58, 39403949.

(73) Rios, L. Y.; Gonthier, M. P.; Remesy, C.; Mila, I.; Lapierre, C.; Lazarus, S. A.; Williamson, G.; Scalbert, A. Chocolate intake increases urinary excretion of polyphenol-derived phenolic acids in healthy human subjects. Am. J. Clin. Nutr. 2003, 77, 912-918.

(74) Aura, A. M.; O’Leary, K. A.; Williamson, G.; Ojala, M.; Bailey, M.; Puupponen-Pimiä, R.; Nuutila, A. M.; Oksman-Caldentey, K. M.; Poutanen, K. Quercetin derivatives are deconjugated and converted to hydroxyphenylacetic acids but not methylated by human fecal flora in vitro. J Agric Food Chem 2002, 50, 1725-1730.

(75) Boudonck, K. J.; Mitchell, W.; Német, L.; Keresztes, L.; Nyska, A.; Shinar, D.; Rosenstock, M. Discovery of Metabolomics Biomarkers for Early Detection of Nephrotoxicity. Toxicol. Pathol. 2009, 37, 280-292. 
(76) Wishart, D. S.; Jewison, T.; Guo, A. C.; Wilson, M.; Knox, C.; et al., HMDB 3.0 - The Human Metabolome Database in 2013. Nucleic Acids Res. 2013, Jan 1;41(D1):D8017. 23161693

(77) Fontana, M.; Giovannitti, F.; Pecci, L. The protective effect of hypotaurine and cysteine sulphinic acid on peroxynitrite-mediated oxidative reactions. Free Radic Res 2008, 42, 320-330.

(78) Porter, F. D.; Scherrer, D. E.; Lanier, M. H.; Langmade, S. J.; Molugu, V.; Gale, S. E.; Olzeski, D.; Sidhu, R.; Dietzen, D. J.; Fu, R.; Wassif, C. A.; Yanjanin, N. M.; Marso, S. P.; House, J.; Vite, C.; Schaffer, J. E.; Ory, D. S. Cholesterol oxidation products are sensitive and specific blood-based biomarkers for Niemann-Pick C1 disease. Science translational medicine 2010, 2, 56ra81.

(79) Ribas, G. S.; Pires, R.; Coelho, J. C.; Rodrigues, D.; Mescka, C. P.; Vanzin, C. S.; Biancini, G. B.; Negretto, G.; Wayhs, C. A.; Wajner, M.; Vargas, C. R. Oxidative stress in NiemannPick type C patients: a protective role of $\mathrm{N}$-butyl-deoxynojirimycin therapy. Int. J. Dev. Neurosci. 2012, 30, 439-444.

(80) Sakuragawa, T.; Hishiki, T.; Ueno, Y.; Ikeda, S.; Soga, T.; Yachie-Kinoshita, A.; Kajimura, M.; Suematsu, M. Hypotaurine is an Energy-Saving Hepatoprotective Compound against Ischemia-Reperfusion Injury of the Rat Liver. J Clin Biochem Nutr 2010, 46, 126-134.

(81) Liu, R. M.; Gaston Pravia, K. A. Oxidative stress and glutathione in TGF-beta-mediated fibrogenesis. Free Radic. Biol. Med. 2010, 48, 1-15. 
(82) Cabre, M.; Camps, J.; Paternain, J. L.; Ferre, N.; Joven, J. Time-course of changes in hepatic lipid peroxidation and glutathione metabolism in rats with carbon tetrachloride-induced cirrhosis. Clin. Exp. Pharmacol. Physiol. 2000, 27, 694-699.

(83) Lu, S. C. Regulation of glutathione synthesis. Mol. Aspects Med. 2009, 30, 42-59.

(84) Lehninger, A. L.; Nelson, D.L.; Cox, M. M. Principles of Biochemistry. Worth Publishers; New York: 1993.

(85) Liaudet, L.; Mabley, J. G.; Soriano, F. G.; Pacher, P.; Marton, A.; Haskó, G.; Szabó, C. Inosine reduces systemic inflammation and improves survival in septic shock induced by cecal ligation and puncture. Am J Respir Crit Care Med. 2001, 164(7), 1213-1220.

(86) Banerjee, R. Redox Biochemistry. John Wiley \& Sons; Hoboken, New Jersey: 2008.

(87) Boenzi, S.; Deodato, F.; Taurisano, R.; Martinelli, D.; Verrigni, D.; Carrozzo, R.; et al. A new simple and rapid LC-ESI-MS/MS method for quantification of plasma oxysterols as dimethylaminobutyrate esters. Its successful use for the diagnosis of Niemann-Pick type C disease. Clin Chim Acta. 2014, 437, 93-100.

(88) Cluzeau, C. V.; Watkins-Chow, D. E.; Fu, R.; Borate, B.; Yanjanin, N.; Dail, M. K.; et al. Microarray expression analysis and identification of serum biomarkers for Niemann-Pick disease, type C1. Hum. Mol. Genet. 2012, 21(16), 3632-46.

(89) Sheth, J. J.; Sheth, F. J.; Oza, N. J.; Gambhir, P. S.; Dave, U. P.; Shah, R. C. Plasma chitotriosidase activity in children with lysosomal storage disorders. Indian J Pediatr. 2010, 77(2), 203-205. 
(90) Welford, R. W.; Garzotti, M.; Marques Lourenco, C.; Mengel E, Marquardt, T.; Reunert, J.; et al. Plasma lysosphingomyelin demonstrates great potential as a diagnostic biomarker for Niemann-Pick disease type C in a retrospective study. PLoS One. 2014, 9(12), e114669.

(91) Giese, A.K.; Mascher, H.; Grittner, U.; Eichler, S.; Kramp, G.; Lukas. J.; et al. A novel, highly sensitive and specific biomarker for Niemann-Pick type C1 disease. Orphanet J Rare Dis. 2015 10, 78,015-0274-1.

(92) Maekawa, M.; Misawa, Y.; Sotoura, A.; Yamaguchi, H.; Togawa, M.; Ohno, K. et al. LC/ESI-MS/MS analysis of urinary 3beta-sulfooxy-7beta-N-acetylglucosaminyl-5-cholen-24oic acid and its amides: new biomarkers for the detection of Niemann-Pick type C disease. Steroids 2013, 78(10), 967-972.

(93) Vanier, M. T.; Gissen, P.; Bauer, P.; Coll, M. J.; Burlina, A.; Hendriksz, C. J.; Latour, P.; Goizet, C.; Welford, R. W. D.; Marquardt, T.; Kolb, S. A. Diagnostic tests for Niemann-Pick disease type C (NP-C): A critical review. Mol. Genet. Metab. 2016, http://dx.doi.org/10.1016/j.ymgme.2016.06.004 


\section{Figure Legends}

Figure 1. ${ }^{1} \mathrm{H}-\mathrm{NMR}$ spectra of an aqueous extract of an NP-C1 disease liver biopsy sample from a female mouse collected at 9 weeks. A typical spectrum is shown. Abbreviations: 1, Unassigned-1; 2, Leu- $\mathrm{CH}_{3} / \mathrm{Ile}-\mathrm{C} 5-\mathrm{CH}_{3} / \mathrm{Val}-\mathrm{CH}_{3 \mathrm{a}} ; 3,2-\mathrm{Aminobutyrate}-\mathrm{CH}_{3} ; 4$, Val- $\mathrm{CH}_{3 \mathrm{~b}} ; 5$, Propylene glycol- $\mathrm{CH}_{3} ; 6$, Lactate- $\mathrm{CH}_{3} ; 7$, Alanine- $\mathrm{CH}_{3} ; 8$, Lys- $\mathrm{C} 5-\mathrm{CH}_{2} / \mathrm{Orn}-\mathrm{C}_{4}-\mathrm{CH}_{2} ; 9$, Acetate$\mathrm{CH}_{3} ; 10$, Acetonitrile; 11 , Met- $\mathrm{CH}_{3} ; 12$, GSSH-Glu-C3-CH$/ \mathrm{Gln}-\mathrm{C}_{3}-\mathrm{CH}_{2} ; 13$, Glu-C4-CH$; 14$, Oxalacetate- $\mathrm{CH}_{2} ; 15$, Succinate- $\mathrm{CH}_{2} ; 16$, Gln-C4- $\mathrm{CH}_{2} ; 17$, Met- $\mathrm{C} 4-\mathrm{CH}_{2} /$ Hypotaurine- $\mathrm{C} 4-\mathrm{CH}_{2}$; 18 , Asp- $\mathrm{CH}_{2 \mathrm{a}} ; 19$, Sarcosine- $\mathrm{CH}_{3} ; 20$, Asp- $\mathrm{CH}_{2 \mathrm{~b}} ; 21, \mathrm{TMA}-\mathrm{CH}_{3^{\prime} \text { s }} ; 22$, Dimethylglycine- $\mathrm{CH}_{3^{\prime} \text { s; }} ; 23$, GSSH-Cys-C3-CH $\mathrm{CH}_{2} ; \quad 24$, Creatine- $\mathrm{CH}_{3} ; \quad 25$, Lys-C6- $-\mathrm{CH}_{2} ; \quad 26$, Orn- $\mathrm{C} 5-\mathrm{CH}_{2} ; \quad 27$, Choline/Phosphocholine- $\mathrm{CH}_{3^{\prime} \mathrm{s}} ; \quad 28$, Glycerophosphocholine- $\mathrm{CH}_{3}{ }^{\prime} \mathrm{s} ; 29$, Taurine- $\mathrm{S}-\mathrm{CH}_{2} / \beta-$ Glucose-C2-CH; 30, Methanol; 31, $\beta / \alpha$-Glucose-C4-CH; 32, Taurine- $\mathrm{N}-\mathrm{CH}_{2} ; 33, \beta$-Glucose$\mathrm{C} 3 / 5-\mathrm{CH} ; 34$, Glycine; 35, Glycerol-C1/3- $\mathrm{CH}_{2} ; 36, \beta-G l u c o s e-C 6-\mathrm{CH}_{\mathrm{a}} / \alpha-\mathrm{Glucose}-\mathrm{C} 3-\mathrm{CH} / 6-\mathrm{CH}_{2}$; $37, \beta$-Glucose- $\mathrm{C} 6-\mathrm{CH}_{\mathrm{b}} ; 38$, Betaine- $\mathrm{CH}_{2} ; 39$, Lactate- $\mathrm{CH}_{2} ; 40$, Thr- $\mathrm{CH}_{2} ; 41$, Ascorbate; $42, \mathrm{GSH}-$ Cys-CH; 43, $\beta$-Glucose/Glucose-6P-C1-CH; 44, Phosphoenolpyruvate- $\mathrm{CH} 2 \mathrm{a} ; \quad 45, \quad \alpha-$ Glucose/Glucose-6P-C1-CH; 46, Phosphoenolpyruvate- $\mathrm{CH}_{2 \mathrm{~b}} / \mathrm{Glycogen;} 47$, Uracil-CO-CH; 48, GTP-C1'-CH; 49, 3-Hydroxyphenylacetate- $\mathrm{CH} 2 ; 50$, Tyr-C2/6-CH; 51, Tyr- $\mathrm{C} 3 / 5-\mathrm{CH} ; 52,3-$ Hydroxyphenylacetate- $\mathrm{C} 4 / 6-\mathrm{CH} ; 53$, Phenylalanine; 54, Uracil- $\mathrm{NH}-\mathrm{CH} ; 55$, Niacinamide-C5$\mathrm{CH}$; 56, Guanosine-C8-CH; 57, GTP/GMP-C8-CH; 58, GMP-C8-CH/Hypoxanthine-C2-CH; 59, Hypoxanthine-C8-CH; 60, Nicotinate- $\mathrm{C} 4-\mathrm{CH} ; \quad 61$, Nicotinate/Niacinamide-C6-CH; 62, Nicotinate/Niacinamide-C2-CH.

Figure 2. (a) Three-Dimensional (3D) PC4 vs. PC3 vs. PC2 scores plot arising from PCA of the preliminary (complete) dataset with $\mathrm{P}=136$ ISB predictor $(\mathrm{X})$ variables. Key: WT, red squares; NP-C1, yellow diamonds; and HET, green circles. (b) Multidimensional scaling plot 
of a random forest proximity matrix from HET/WT vs NP-C1 analysis demonstrating the success of discrimination from a single RFs iteration.

Figure 3. (a) Three-Dimensional (3D) component 3 vs. component 2 vs. component 1 scores plot arising from PLS-DA of the liver sample dataset incorporating only the 3- and 9-week time-point samples for both the NP-C1 and HET/WT classifications [with $\mathrm{P}=136$ ISB predictor (X) variables]; (b) O-PLS-DA plot of orthogonal T score vs. T score for this 3- and 9week time-point dataset; (c) Permutation testing plot for the $R^{2} Y$ and $Q^{2}$ values determined from this analysis.

Figure 4. Box plots of TSP-normalised ISB resonance intensities of the 12 metabolites identified as discriminatory variables by RFs analysis vs. time-point (weeks) for the NP-C1 (grey) and HET/WT (white) phenotypic classifications.

Figure 5. (a) ROC curves for the RFs models developed. Combinations of variables gave rise to AUROC values ranging from 0.801 for 2 variables, to 0.941 with 12 variables, as indicated in the lower right-hand inset. (b) Predictive accuracies as a function of increasing numbers of biomarker variables employed for constructing the RFs model.

Figure 6. (a) 2D PCA scores plot of PC2 versus PC1 (which account for a cumulative variance of $70.6 \%$ ) for a model incorporating only the most significant RFs-determined 12 biomarker metabolites; (b) Corresponding loadings plot for these biomarkers for the combined NP-C1 and HET/WT dataset: glutamate, hypotaurine/methionine, valine, lysine/ornithine, tyrosine and phenylalanine all had highly positive PC1 and weakly negative PC2 loadings, whereas 
nicotinate/niacinamide, inosine, 3-hydroxyphenylacetate and the unassigned $\delta=0.77-0.80$ ppm resonance had highly positive PC2 with low or intermediately-positive PC1 ones.

Figure 7. Venn diagram showing similarities and differences in biomarker candidates (detected in NP-C1 disease mutant mouse model liver, human and mutant mouse model blood plasma/serum, and human urine) between this study and those previously reported. ${ }^{14,49,78,87-93}$. Abbreviations: Lyso-SM-509, lyso-sphingomyelin-509; MCs, monohexosylceramides; LGALS3, galectin-3; CTSD, cathepsin D; 3-AIB, 3-aminoisobutyrate; 3-HPA, 3-hydroxyphenylacetate; PEP, phosphoenolpyruvate; 2PY and 4PY, N-methyl-2pyridone-5-carboxamide and $\mathrm{N}$-methyl-4-pyridone-5-carboxamide respectively; ${ }^{*}$ this study. 

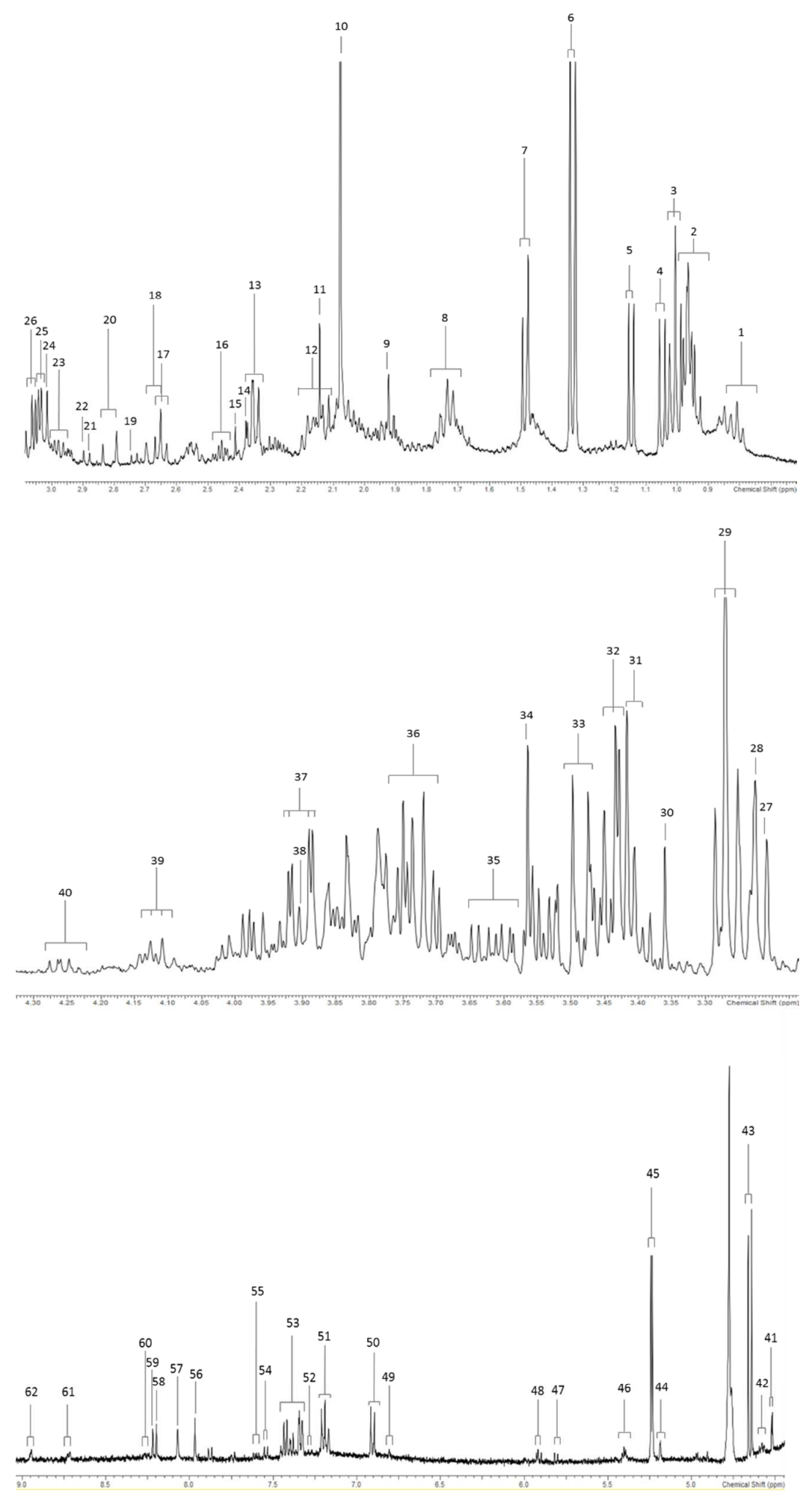

Figure 1. 
(a) $\quad \mathrm{WT} \quad \mathrm{HET} \diamond \mathrm{NPC}$

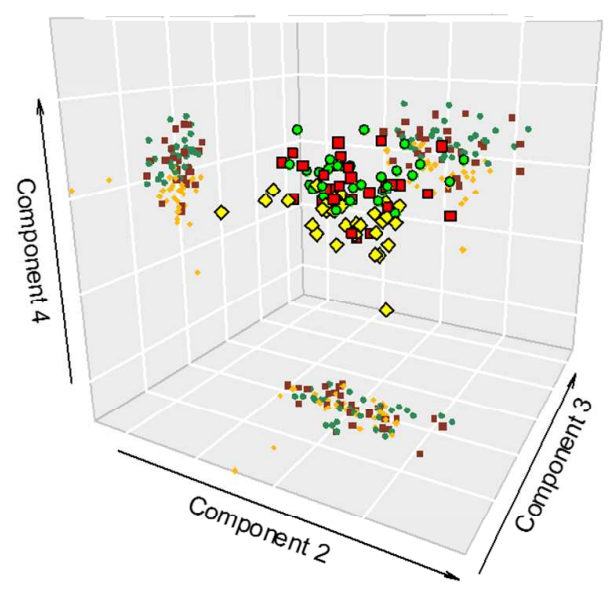

(b) $\quad \mathrm{HET} / \mathrm{WT} \quad \diamond \mathrm{NPC}$

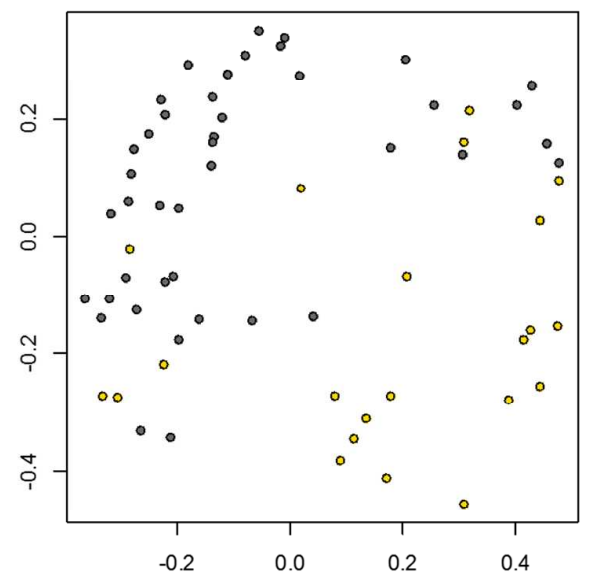

Figure 2. 


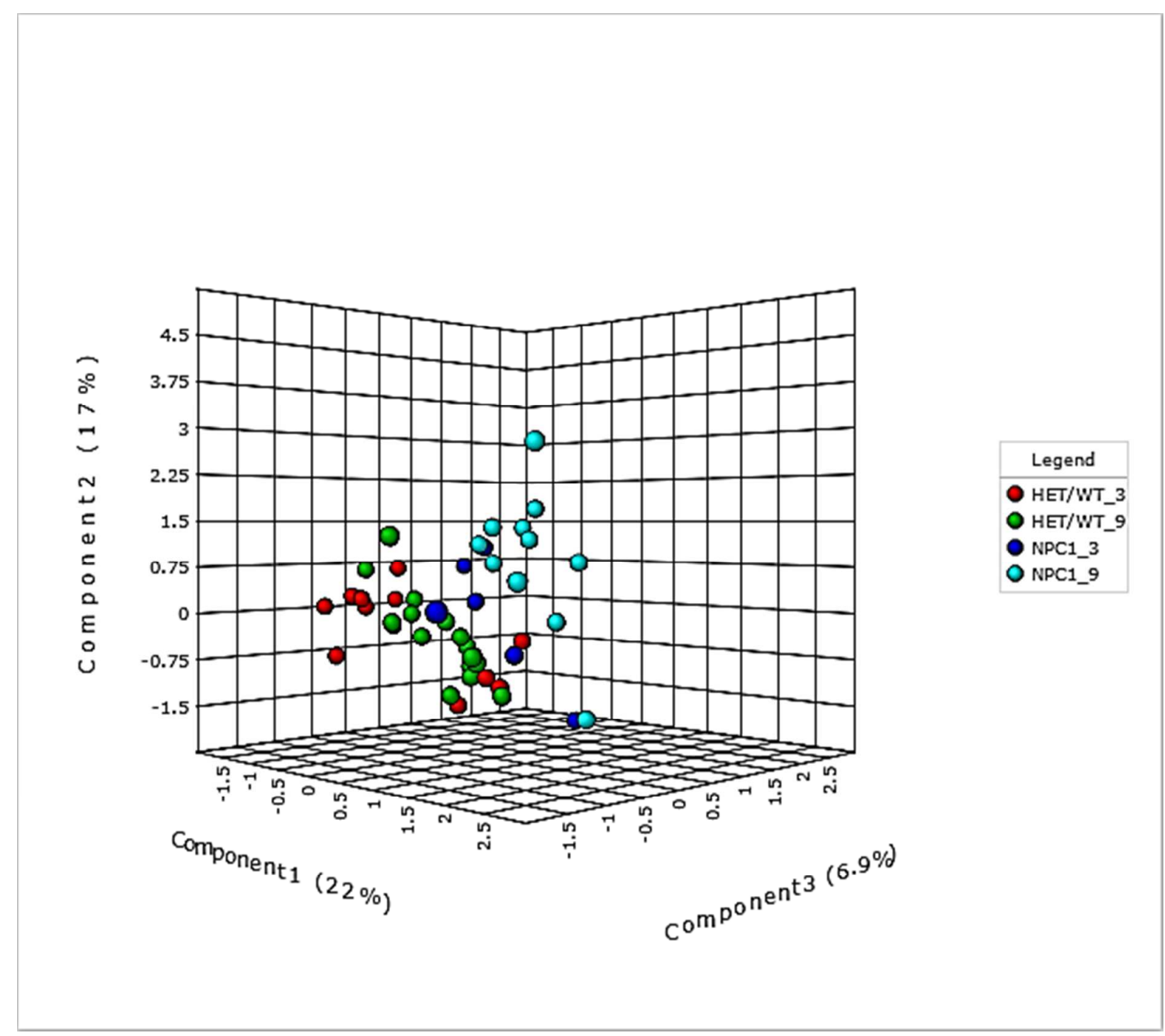

Figure 3(a) 


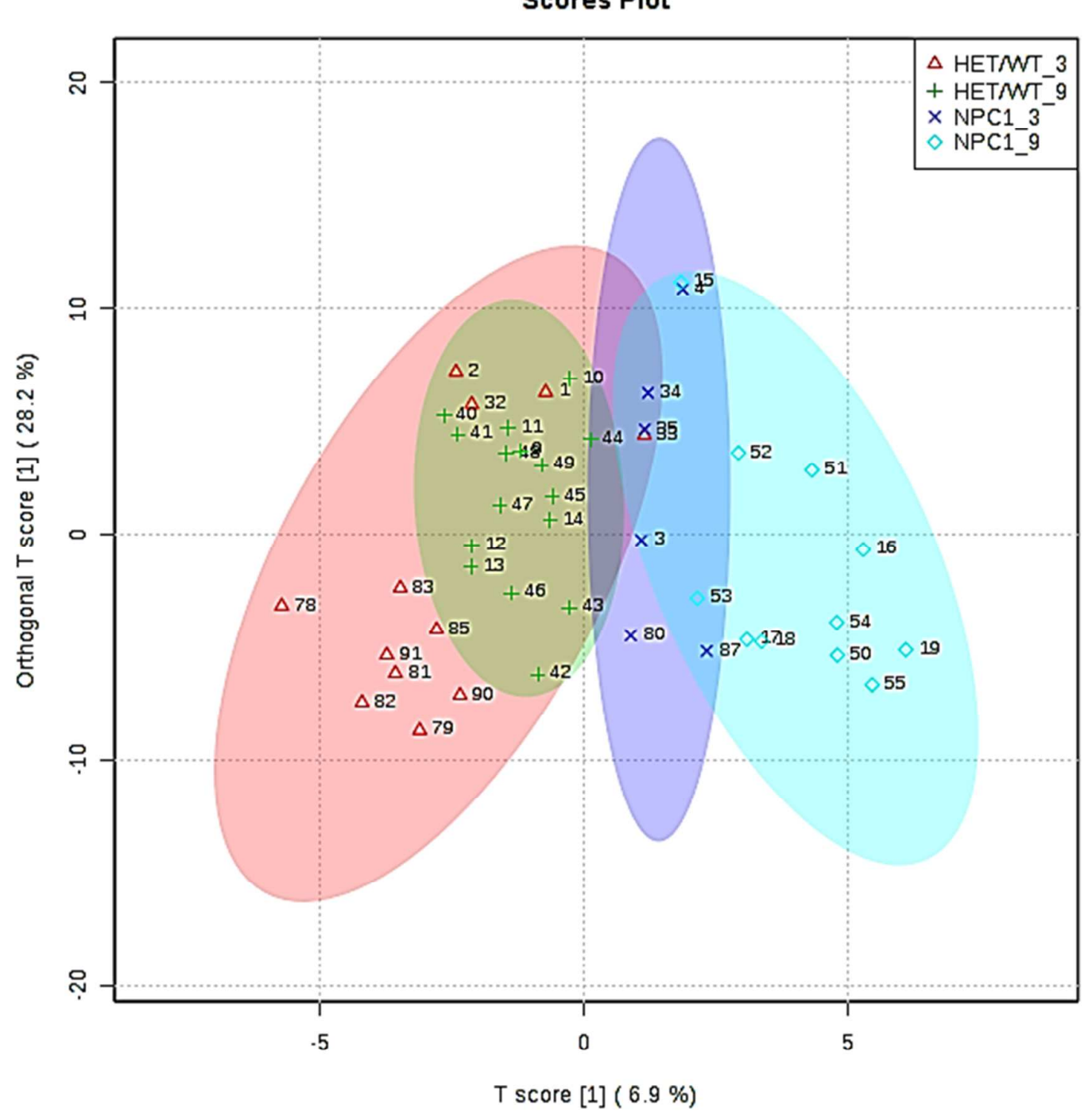

Figure 3(b) 


\section{Page 59 of 65}

Journal of Proteome Research

1
2
3
4
5
6
7
8
9
10
11
12
13
14
15
16
17
18
19
20
21
22
23
24
25
26
27
28
29
30
31
32
33
34
35
36
37
38
39
40
41
42
43
44
45
46
47
48
49
50
51
52
53
54
55
56
57
58
60

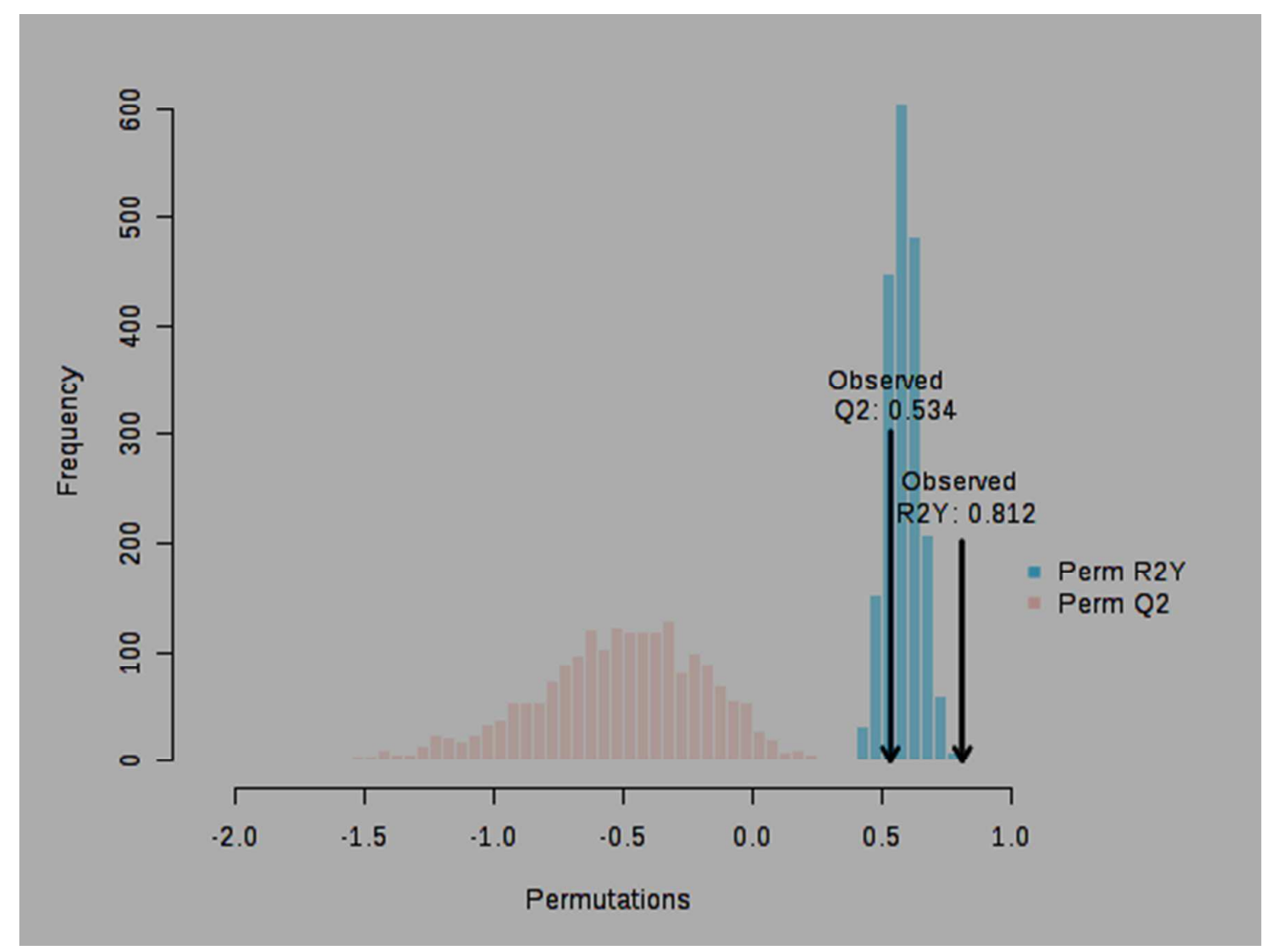

Figure $3(c)$ 
HET/WT NPC
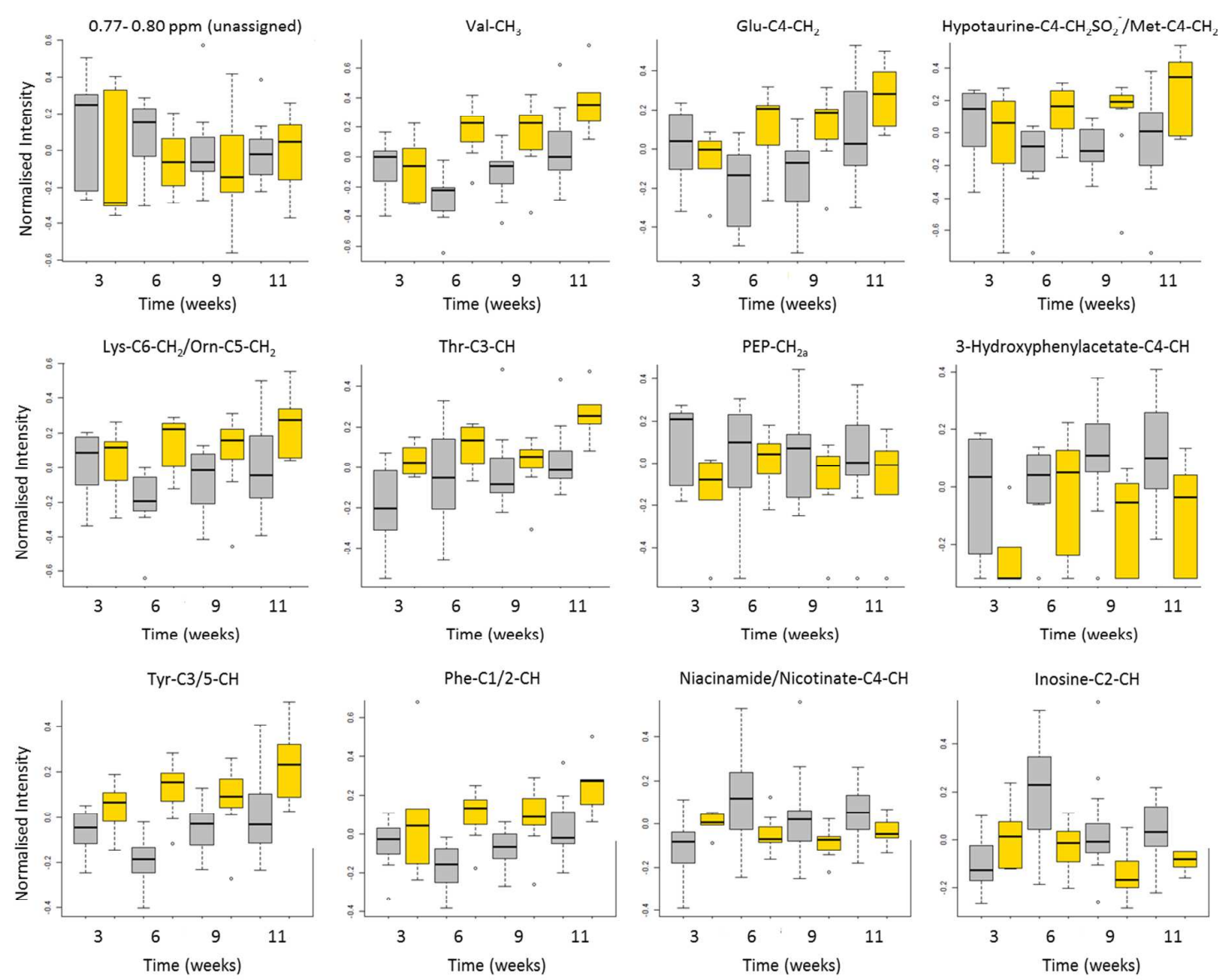

Niacinamide/Nicotinate-C4-CH
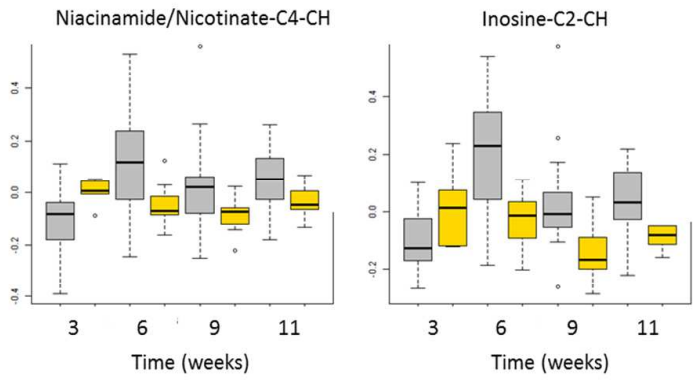

Figure 4.

(a)

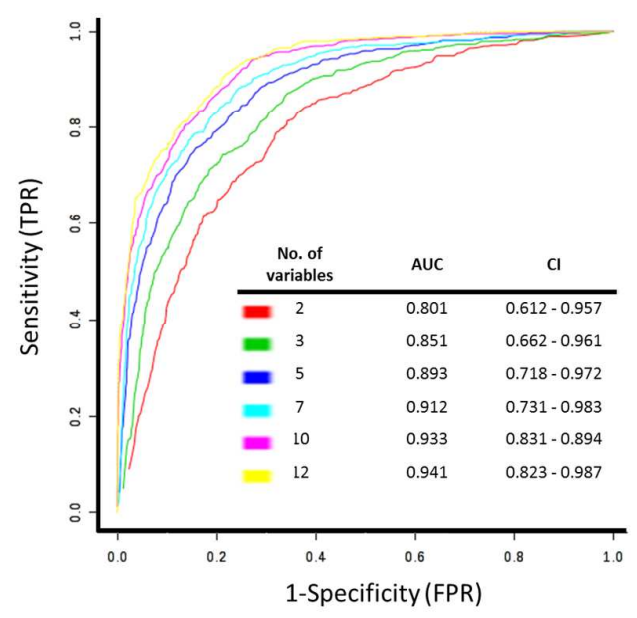

(b)

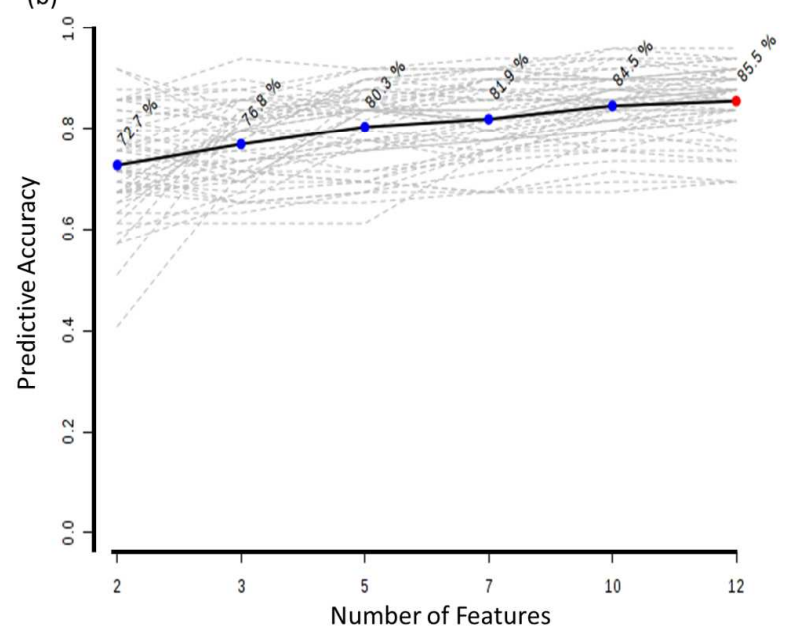

Figure 5. 


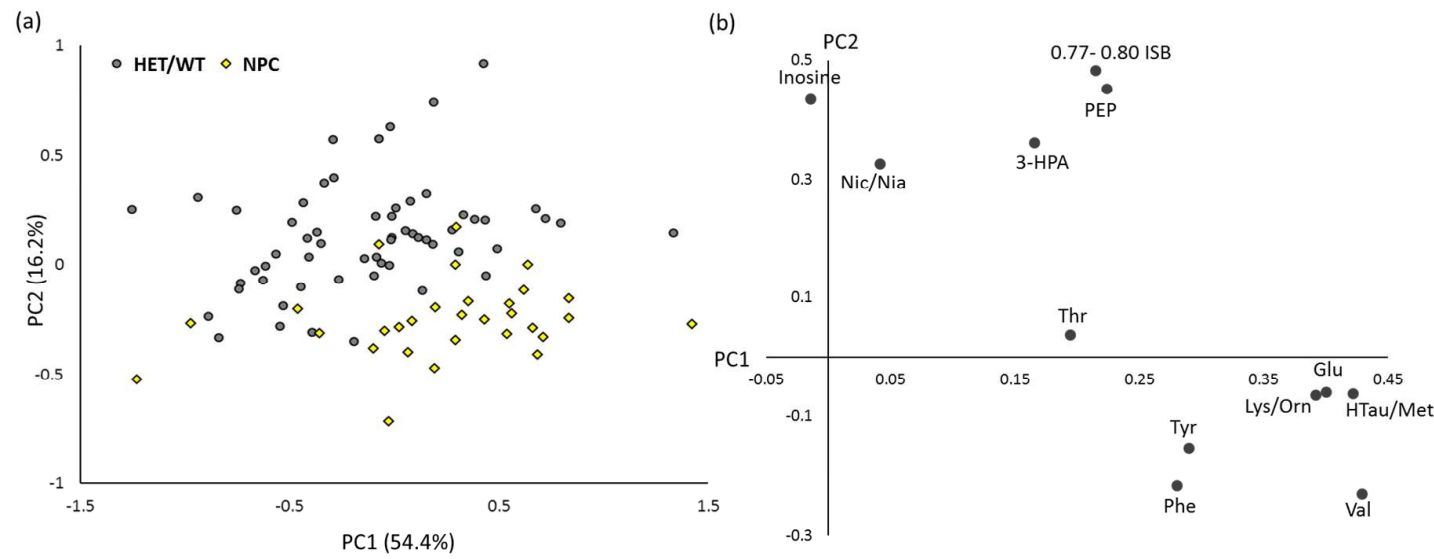

Figure 6. 
3-AIB $(\uparrow)^{14} ; 3$-HIB $(\uparrow)^{14}$; Bile acids $(\uparrow)^{14}$ Quinolinate $(\uparrow)^{14}$; Ile $(\uparrow)^{14}$; Trigonelline $(\downarrow)^{14}$; NMN amide $(\downarrow)^{14} ; \operatorname{Gln}^{14}(\uparrow)$; Ala $(\uparrow)^{14} ; 2$ NyN amide $(\downarrow)$, Gla 14 Hydroxyglutamate $(\downarrow)^{14} ; 2$-Hydroxy-3-
methylbutyrate $(\downarrow)^{14} ;$ Nicotinamide riboside methylbutyrate $(\downarrow)^{14}$; Nicotinamide riboside
$(\uparrow)^{14} ; 2 \mathrm{PY}(\downarrow)^{14} ; 4 \mathrm{PY}(\downarrow)^{14} ; 3$-Methylhistidine $(\uparrow)^{14}$; TMA $(\uparrow)^{14}$; Suc $(\uparrow)^{14}$; Pyroglutamate $(\uparrow)^{14}$ N-Acetylsugars $(\uparrow)^{14}$; Propane-1,2-diol $(\uparrow)^{14} ; 3 \beta$ Sulfoxy-7 $\beta-\mathrm{N}$-acetylglucosaminyl-5-cholen-24-

oate and its glycine and taurine conjugates $(\uparrow)^{92}$,

$3 \beta, 7 \beta$-Dihydroxy-5-cholen-24-oate and its glycine and taurine conjugates $(\uparrow)^{93}$

Figure 7. MOUS MODEL AND HUMAN):

7-Ketocholesterol $(\uparrow)^{78,87} ; 3 \beta, 5 \alpha, 6 \beta-C h l o e s t a n e-t r i o l ~(\uparrow)^{78,87}$; LALS3 $(\uparrow)^{88}$; CTSD $(\uparrow)^{88}$; Chitotriosidase $(\uparrow)^{89}$; $(\uparrow)^{49}$

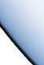

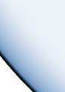
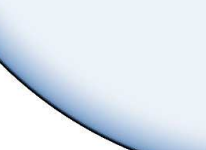
Nicotinate $(\downarrow)^{14}$ LIVER (NP-C1 MUTANT MOUSE MODEL):

Phe $(\uparrow)^{*}$; Tyr $(\uparrow)^{*}$; HTau/Met $(\uparrow)^{*} ;$ 3-HPA $(\downarrow)^{*}$; Lys/Orn $(\uparrow)^{*} ;$ PEP $(\downarrow)^{*} ;$ Thr $(\uparrow)^{*}$; Glu $(\uparrow)^{*} ;$ Lys/Orn $(\uparrow)^{*}$; PEP $(\downarrow)^{*}$; Thr $(\uparrow)^{*}$; Glu $(\uparrow)^{*}$;
Inosine $(\downarrow)^{*}$; Niacinamide/Nicotinate $(\downarrow)^{*}$; GM2 
Tables

\begin{tabular}{|c|c|c|c|c|c|c|c|c|c|c|c|c|}
\hline \multirow[b]{2}{*}{ Disease Class } & \multicolumn{12}{|c|}{ Collection Time-Point (weeks) } \\
\hline & 3 & 6 & 9 & 11 & 3 & 6 & 9 & 11 & 3 & 6 & 9 & 11 \\
\hline WT & 3 & 3 & 3 & 3 & 3 & 3 & 5 & 5 & 6 & 6 & 8 & 8 \\
\hline HET & 3 & 3 & 4 & 5 & 3 & 3 & 5 & 5 & 6 & 6 & 9 & 10 \\
\hline \multirow[t]{2}{*}{ NP-C1 } & 3 & 3 & 5 & 3 & 3 & 4 & 6 & 3 & 6 & 7 & 11 & 6 \\
\hline & \multicolumn{4}{|c|}{ Female } & \multicolumn{4}{|c|}{ Male } & \multicolumn{4}{|c|}{ Total } \\
\hline
\end{tabular}

Table 1. Liver samples available for ${ }^{1} \mathrm{H}$ NMR-linked metabolomics analysis at time-points of $3,6,9$ and 11 weeks.

\begin{tabular}{|c|c|c|c|c|c|}
\hline $\begin{array}{l}\text { Variable } \\
\text { Ranking }\end{array}$ & ISB (ppm) & Multiplicity & Assignment & $\begin{array}{l}\text { Fold } \\
\text { change }\end{array}$ & $\begin{array}{l}\text { 'Between-disease } \\
\text { classifications' } \\
\text { ANCOVA p-value }\end{array}$ \\
\hline 1 & 7.30-7.35 & $d(6.8)$ & Phenylalanine- $\mathrm{C} 1 / \mathrm{C} 2-\mathrm{CH}$ & 1.74 & $2.73 \times 10^{-7}$ \\
\hline 2 & 7.17-7.23 & $d(8.6)$ & Tyrosine- $\mathrm{C} 3 / \mathrm{C} 5-\mathrm{CH}$ & 1.58 & $9.48 \times 10^{-8}$ \\
\hline 3 & 7.35-7.39 & $m$ & Phenylalanine- $\mathrm{C} 3 / 4 / 5-\mathrm{CH}$ & 3.49 & $9.63 \times \times 10^{-4}$ \\
\hline 4 & $0.94-1.00$ & $d(7.1)$ & Valine- $\mathrm{CH}_{3}$ & 1.54 & $1.22 \times 10^{-6}$ \\
\hline 5 & $2.62-2.67$ & $m$ & Hypotaurine- $\mathrm{C} 4-\mathrm{CH}_{2} \mathrm{SO}_{2}^{-} /$Methionine- $\mathrm{C} 4-\mathrm{CH}_{2}$ & 1.87 & $1.48 \times \times 10^{-3}$ \\
\hline 6 & $6.78-6.81$ & $m$ & 3-Hydroxyphenylacetate-C4-CH & -2.94 & $5.78 \times 10^{-6}$ \\
\hline 7 & 2.99-3.05 & $t / t$ & Lysine-C6- $\mathrm{CH}_{2} /$ Ornithine-C5- $\mathrm{CH}_{2}$ & 1.49 & $6.91 \times 10^{-5}$ \\
\hline 8 & $6.86-6.93$ & $d(8.2)$ & Tyrosine- $\mathrm{C} 2 / \mathrm{C} 6-\mathrm{CH}$ & 2.05 & $1.01 \times 10^{-3}$ \\
\hline 9 & $5.16-5.21$ & $t(1.1)$ & PEP-CH $\mathrm{CH}_{2 \mathrm{a}}$ & -1.74 & 0.027 \\
\hline 10 & $0.77-0.80$ & $t(7.5)$ & Unassigned & -1.27 & 0.055 \\
\hline 11 & $4.24-4.30$ & $m$ & Threonine- $\mathrm{C} 3-\mathrm{CH}$ & 1.32 & $2.95 \times 10^{-4}$ \\
\hline 12 & $2.32-2.40$ & $m$ & Glu-C4-CH & 1.38 & $1.032 \times 10^{-3}$ \\
\hline 13 & $1.68-1.74$ & $m / m$ & Lysine-C5- $\mathrm{CH}_{2} /$ Ornithine-C4- $\mathrm{CH}_{2}$ & 1.42 & $1.21 \times 10^{-4}$ \\
\hline 14 & $8.32-8.36$ & $s$ & Inosine- $\mathrm{C} 2-\mathrm{CH}$ & -1.75 & $2.86 \times 10^{-3}$ \\
\hline 15 & $8.23-8.29$ & $d d$ & Nicotinate/Niacinamide-C4-CH & -1.28 & 0.037 \\
\hline
\end{tabular}

Table 2. Key ${ }^{1} \mathrm{H}$ NMR ISB biomarker variable features derived from application of MV RFs analysis: ISB variables are ranked from 1 to 15 based on their MDA values. 'Between-disease classifications' ANCOVA $p$ values and fold-changes are also provided. ${ }^{1} \mathrm{H}$ NMR resonances, their coupling patterns and assignments were confirmed via the methods outlined in section 2.4. Positive fold change values indicate upregulations in NP-C1 disease, whereas negative ones indicate a higher level determined in the HET/WT group. 


\begin{tabular}{|c|c|c|c|}
\hline Metabolite & $\begin{array}{l}\text { Upregulation }(\uparrow) \\
\text { or } \\
\text { Downregulation } \\
(\downarrow) \text { : this study }\end{array}$ & $\begin{array}{l}\text { Biomarker status in } \\
\text { Fibrosis-Cirrhosis } \\
\text { (Upregulation or } \\
\text { Downregulation) }\end{array}$ & $\begin{array}{l}\text { Biomarker Status in } \\
\text { Further Liver } \\
\text { Disease/ } \\
\frac{\text { Toxicological }}{\text { Damage }^{43}}\end{array}$ \\
\hline Glutamate & $\uparrow$ & Yes (个) & Yes (个) \\
\hline Hypotaurine & $\uparrow$ & No & No \\
\hline Methionine & $\uparrow$ & Yes (个) & No \\
\hline Threonine & $\uparrow$ & No & Yes (个) \\
\hline Lysine & $\uparrow$ & No & No \\
\hline Ornithine & $\uparrow$ & No & No \\
\hline Valine & $\uparrow$ & No & Yes (个) \\
\hline Phenylalanine & $\uparrow$ & Yes (个) & Yes (个) \\
\hline Tyrosine & $\uparrow$ & Yes (个) & Yes (个) \\
\hline 3-Hydroxyphenylacetate & $\downarrow$ & No & No \\
\hline Phosphoenolpyruvate & $\downarrow$ & No & $n-d$ \\
\hline Inosine & $\downarrow$ & Yes (个) & $n-d$ \\
\hline Niacinamide & $\downarrow$ & Yes $(\downarrow)$ & $n-d$ \\
\hline Nicotinate & $\downarrow$ & No & $n-d$ \\
\hline
\end{tabular}

Table 4. Summary of the biomarker status of the significant predictor metabolite variables detected in this investigation in fibrosis-cirrhosis development ${ }^{33-35}$, and also in further liver disease/hepatic toxicity ${ }^{43}$ investigations reported. Abbreviations: $n-d$, not determined. 
NP-C1 Mutant (Npc1/-; NP-C1) Mouse

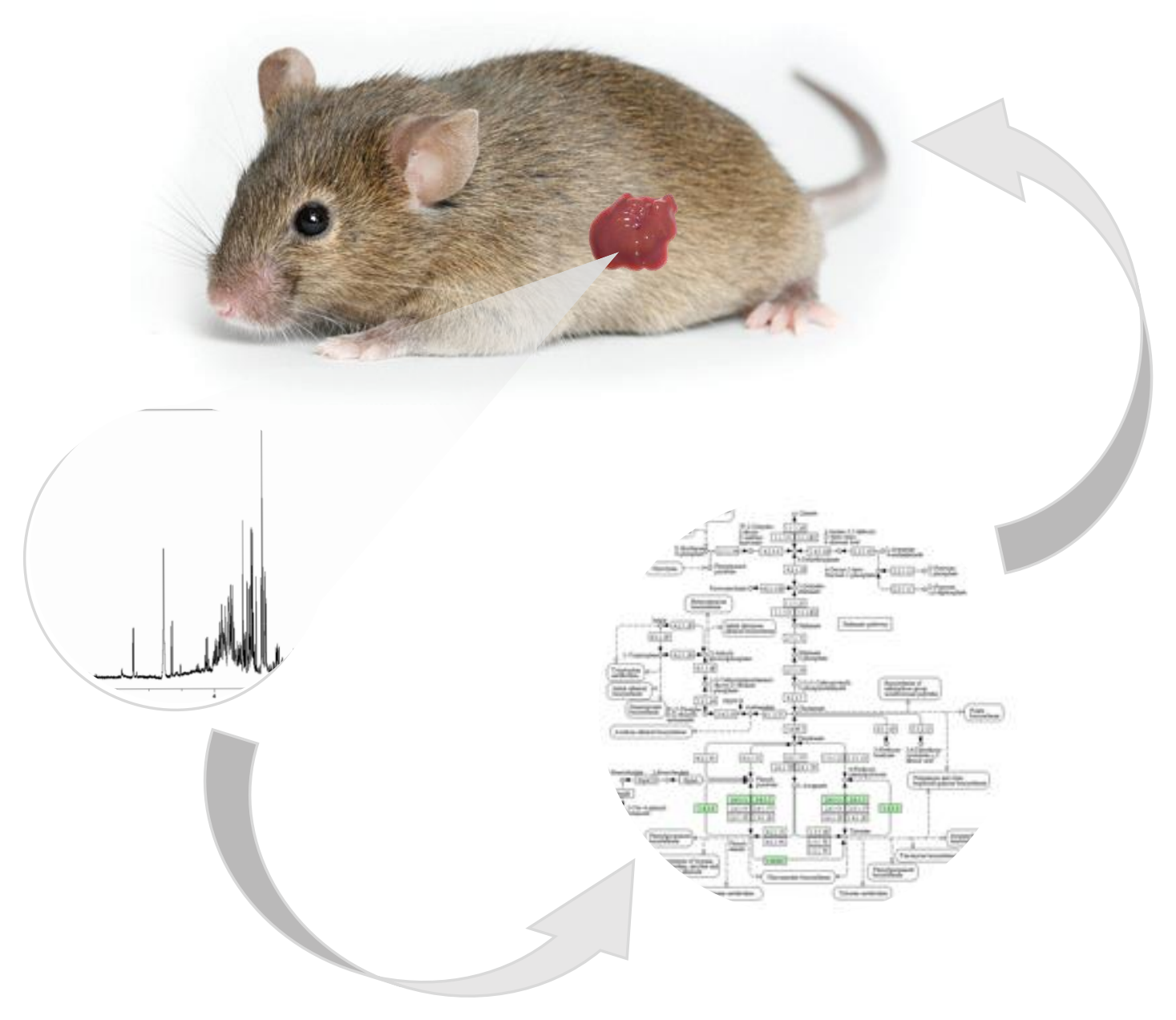

ACS Paragon Plus Environment 